\title{
Flow networks: A characterization of geophysical fluid transport
}

\author{
Enrico Ser-Giacomi, Vincent Rossi, Cristóbal López, and Emilio Hernández-García \\ Instituto de Física Interdisciplinar y Sistemas Complejos, IFISC (CSIC-UIB), Campus Universitat de les Illes \\ Balears, E-07122 Palma de Mallorca, Spain
}

(Received 5 September 2014; accepted 2 February 2015; published online 12 February 2015)

\begin{abstract}
We represent transport between different regions of a fluid domain by flow networks, constructed from the discrete representation of the Perron-Frobenius or transfer operator associated to the fluid advection dynamics. The procedure is useful to analyze fluid dynamics in geophysical contexts, as illustrated by the construction of a flow network associated to the surface circulation in the Mediterranean sea. We use network-theory tools to analyze the flow network and gain insights into transport processes. In particular, we quantitatively relate dispersion and mixing characteristics, classically quantified by Lyapunov exponents, to the degree of the network nodes. A family of network entropies is defined from the network adjacency matrix and related to the statistics of stretching in the fluid, in particular, to the Lyapunov exponent field. Finally, we use a network community detection algorithm, Infomap, to partition the Mediterranean network into coherent regions, i.e., areas internally well mixed, but with little fluid interchange between them. (C) 2015 AIP Publishing LLC. [http://dx.doi.org/10.1063/1.4908231]
\end{abstract}

Water and air transport are among the basic processes shaping the climate of our planet. Heat and salinity fluxes change sea water density and thus drive the global thermohaline circulation. Atmospheric winds force the ocean motion, and also transport moisture, heat or chemicals, impacting the regional climate. These considerations of geophysical fluid motion suggests viewing fluid transport as a transportation network in which fluid advances along different branches that eventually split and merge. In this paper, we exploit this interpretation of fluid transport as a flow network so that we can use the powerful techniques of modern network theory to better characterize transport, mixing, and dispersion, with examples from ocean flows.

\section{INTRODUCTION}

The last two decades have seen important advances in the Lagrangian description of transport and mixing in fluid flows driven by concepts from dynamical systems theory. Nowadays, the techniques used can be roughly classified as follows. On the one hand, some approaches focus on the geometric objects-lines and surfaces-separating fluid regions with different properties. These geometric objects are often identified with invariant manifolds, ${ }^{1-5}$ and more generally they are known under the name of Lagrangian Coherent Structures. ${ }^{6,7}$ Recent advances identify them as minimally stretching material lines. ${ }^{8}$ On the other hand, another class of algorithms has focussed on computing stretching-like fields in the fluid domain, such as different types of Lyapunov exponents or other Lagrangian descriptors. ${ }^{9-14}$ Ridges or singular lines in such fields turn out to be related, under suitable conditions, to the Lagrangian Coherent Structures of the previous approach, and thus organize the fluid flow. Finally, there is a line of research focussing on the moving fluid regions themselves, the so-called set-oriented methods. ${ }^{15-23}$
The relationships among the different approaches have been discussed in the literature. ${ }^{24-29}$ The geometric approaches are designed to follow specific structures during particular transport events, whereas the coarse-graining inherent to the set-oriented methods makes them useful also to estimate statistical properties in more extended space and time intervals. Stretching-field methods can be used to follow particular events or, by simple averaging, ${ }^{30-32}$ also to characterize dispersion and stirring statistics in large areas or long times.

One of the basic tools in the set-oriented methods is the Perron-Frobenius or transfer operator, which quantifies the amount of fluid transported from some initial region to other ones under time evolution. A discretized version of that operator is a transport matrix indicating which part of the fluid domain is connected with which one, and by what amount of flow. In this matrix form, the transfer operator can be read as an adjacency matrix that defines a transportation or flow network, an analogy that has been recently recognized. ${ }^{33-39}$ Within this network interpretation, the powerful tools of network or graph theory ${ }^{40-43}$ become available to extract information about the transport processes.

Network approaches have been used for geophysical systems in the context of climate networks ${ }^{44-46}$ in which the connections among the different locations represent statistical relationships between climatic time series from these locations, inferred from correlations and other statistical methods. ${ }^{47-50}$ There is some recent work relating the connectivity given by correlations to underlying fluid flow. ${ }^{51}$ In this paper, we analyze directly the network describing the material fluid flow among different locations, which we call flow network or transport network. Among other characteristics, this network is directed, weighted, ${ }^{52}$ spatially embedded, ${ }^{53}$ and time-dependent. ${ }^{54}$ We illustrate the general ideas with an exemplary network derived from a realistic simulation of the surface flow in the Mediterranean sea. Our focus is here on the description with network tools of two important 
aspects of transport, namely, the quantification of dispersion and mixing, and the identification of coherent regions which remain relatively isolated from neighboring fluid. Relationships are drawn with the previous approaches that used the geometric, stretching field, and set-oriented methodologies described above, in particular, with Lyapunov exponents and with almost-invariant sets. In most of the paper, we use the language of "water flow" appropriate to our ocean dynamics example, but our methodology is in fact equally applicable to atmospheric motions, to other fluid-dynamics settings and even to flows in the phase space of more abstract dynamical systems. ${ }^{15,34,35}$

The paper is organized as follows. After this introduction, we describe the basic steps to construct a flow network from fluid velocity data (Sec. II). We apply them to a surface flow field modelled for the Mediterranean sea in Sec. III. The resulting network is studied in Sec. IV A to characterize dispersion and mixing in different regions. Appendix A complements some technical aspects relevant here. In Sec. IVB, we apply the network community detection method Infomap (described in more detail in Appendix B) to identify coherent regions in the sea, well mixed internally but with little exchange among them. The paper finishes with a Conclusions section.

\section{FLOW NETWORK CONSTRUCTION FROM FLUID MOTION}

Since fluid flow is a process occurring in continuous space, a discretization procedure involving a coarse-graining of space is needed to have access to the techniques of network theory. Advantages of the discrete point of view have already been shown in geophysical contexts. ${ }^{17,18,20,22,55}$ Here, we enumerate the steps needed to construct the discrete transport network starting from the continuous flow.

\section{A. Discretization of the fluid domain: Nodes}

Networks are composed of discrete building blocks: nodes. Being fluid flow a continuous system, we need a discretized version of it to give a network representation. To do this, we subdivide the fluid domain of interest in a large number $N$ of boxes, $\left\{B_{i}, i=1, \ldots, N\right\}$ so that network node $j$ represents the fluid box $B_{j}$. Although it is not strictly necessary, we consider here the case in which boxes have the same area (in twodimensional flows) or volume (for three dimensions). Then each box will contain exactly the same amount of fluid.

\section{B. Lagrangian simulation: Links and weights}

To complete the construction of our transport network, we need to establish the connections between nodes (i.e., boxes in the fluid domain). We establish a directional link between two nodes when an exchange of fluid occurred from one to another during a given time interval. The weight of this link will be proportional to the amount of fluid transported. This quantity could be obtained from a Lagrangian point of view by following trajectories of ideal fluid particles and keeping record of their initial and final positions (i.e., starting and ending nodes) during the time interval considered.

More formally, we integrate for a fixed time $\tau$ the equation of motion for each particle, from initial condition $\mathbf{x}_{0}$ at time $t_{0}$ until the final position $\mathbf{x}$ at $t_{0}+\tau$, using a velocity field $\mathbf{v}(\mathbf{x}, t)$. This defines the flow map $\Phi_{t_{0}}^{\tau}$

$$
\mathbf{x}\left(t_{0}+\tau\right)=\Phi_{t_{0}}^{\tau}\left(\mathbf{x}_{0}\right)
$$

which moves around single fluid particles. By considering the action of the flow map on all the points contained in a fluid region $A$, we define the action of $\Phi_{t_{0}}^{\tau}$ on whole sets: $A\left(t_{0}+\tau\right)=\Phi_{t_{0}}^{\tau}\left(A\left(t_{0}\right)\right)$.

\section{Construction of the network adjacency matrix}

Applying the flow map to the discrete boxes, we will have an estimation of the flow among each pair of nodes. More explicitly, given the collection of boxes $\left\{B_{i}, i=1, \ldots, N\right\}$, we represent the transport between them by the discrete version of the Perron-Frobenius operator $\mathbf{P}\left(t_{0}, \tau\right)$, obtained within the Ulam approach, whose matrix elements are given by ${ }^{15-20}$

$$
\mathbf{P}\left(t_{0}, \tau\right)_{i j}=\frac{m\left(B_{i} \cap \Phi_{t_{0}+\tau}^{-\tau}\left(B_{j}\right)\right)}{m\left(B_{i}\right)} .
$$

$m(A)$ is a measure assigned to the set $A$. In our case, it is the amount of fluid it contains, i.e., simply its area or volume. Other measures referring, for example, to heat or salt content could be implemented for future applications. Equation (2) states that the flow from box $B_{i}$ to box $B_{j}$ is the fraction of the contents of $B_{i}$ which is mapped into $B_{j}$. We refer to the figure in Appendix A for a plot of the different sets involved. If a nonuniform distribution of some conserved tracer is initially released in the system such that $\left\{p_{i}\left(t_{0}\right), i=1, \ldots, N\right\}$ is the amount of such tracer in each box $\left\{B_{i}\right\}$ at the initial instant $t_{0}$, the matrix $\mathbf{P}\left(t_{0}, \tau\right)$ gives the evolution of this distribution after a time $\tau$ as $p_{j}\left(t_{0}+\tau\right)=\sum_{i=1}^{N} p_{i}\left(t_{0}\right) \mathbf{P}\left(t_{0}, \tau\right)_{i j}$. Writing the $\left\{p_{i}\right\}$ as row vectors: $p\left(t_{0}+\tau\right)=p\left(t_{0}\right) \mathbf{P}\left(t_{0}, \tau\right)$. A probabilistic interpretation of Eq. (2) is that $\mathbf{P}\left(t_{0}, \tau\right)_{i j}$ is the probability for a particle to reach the box $B_{j}$, under the condition that it started from a uniformly random position within box $B_{i}$. The matrix $\mathbf{P}\left(t_{0}, \tau\right)$ is row-stochastic, i.e., it has nonnegative elements and $\sum_{j=1}^{N} \mathbf{P}\left(t_{0}, \tau\right)_{i j}=1$, but not exactly column stochastic. The quantity $\sum_{i=1}^{N} \mathbf{P}\left(t_{0}, \tau\right)_{i j}$ measures the ratio of fluid present in box $B_{j}$ after a time $\tau$ with respect to its initial content at time $t_{0}$. This ratio will be unity, and the matrix doubly stochastic, if the flow $\mathbf{v}(\mathbf{x}, t)$ is incompressible.

As a standard way to evaluate numerically the matrix in Eq. (2), we apply the Lagrangian map to a large number of particles released uniformly inside each of the boxes $\left\{B_{i}\right.$, $i=1, \ldots, N\}$ (see Fig. 1). The initial number of particles $N_{i}$ in each box, a proxy of the amount of fluid it contains, should be proportional to its measure $m\left(B_{i}\right)$ which, with our choice of equal area or volume, results in seeding the same number of particles in each box. The number of particles transported from box $B_{i}$ to box $B_{j}$ gives an estimation of the flow among 


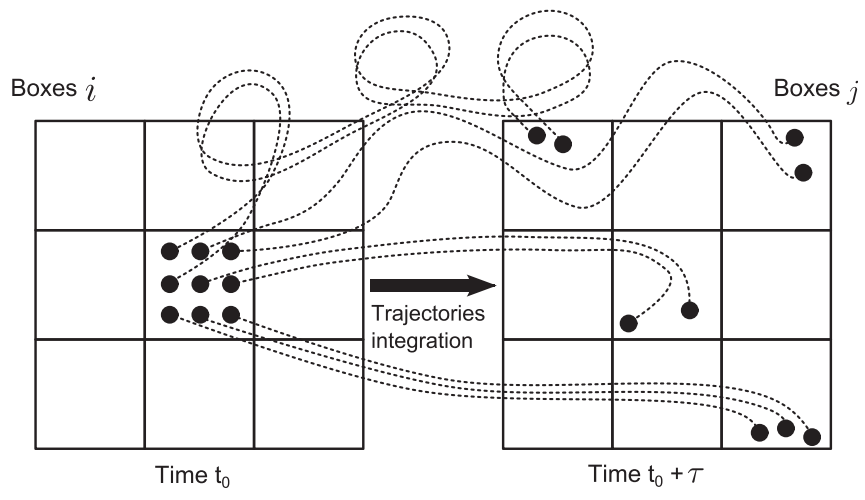

FIG. 1. Transport matrix construction from tracer's advection, following Eq. (3).

these boxes, and a numerical approximation to Eq. (2) is then

$$
\mathbf{P}\left(t_{0}, \tau\right)_{i j} \approx \frac{\text { number of particles from box } i \text { to box } j}{N_{i}}
$$

Because of the time-dependence of the velocity field, the results of the Lagrangian simulations will depend on both the initial time $t_{0}$ and the duration of the simulation $\tau$. Once these parameters are fixed, we can build a network described by a transport matrix $\mathbf{P}\left(t_{0}, \tau\right)$ that characterizes the connections among each pair of nodes from initial time $t_{0}$ to final time $t_{0}+\tau$. We interpret $\mathbf{P}\left(t_{0}, \tau\right)$ as the adjacency matrix of a weighted and directed network so that $\mathbf{P}\left(t_{0}, \tau\right)_{i j}$ is the weight of the link from node $i$ to node $j$.

The network constructed in this way characterizes the final locations of all fluid elements a time $\tau$ after their release at time $t_{0}$, but gives no information on particle locations at intermediate times. Also, since each of the matrices $\mathbf{P}\left(t_{0}+k \tau, \tau\right)$, for $k=0,1, \ldots, n-1$, is a stochastic matrix, one can consider the discrete-time Markov chain in which an initial vector giving occupation probabilities $p\left(t_{0}\right)=\left(p_{1}\left(t_{0}\right)\right.$, $\left.\ldots, p_{N}\left(t_{0}\right)\right)$ for the different boxes is evolved in time as $p\left(t_{n}\right)=p\left(t_{0}\right) \mathbf{P}\left(t_{0}, \tau\right) \mathbf{P}\left(t_{1}, \tau\right) \ldots \mathbf{P}\left(t_{n-1}, \tau\right)$, where $t_{k}=t_{0}+k \tau$. This time evolution will not be exactly equal to the true evolution $p\left(t_{n}\right)=p\left(t_{0}\right) \mathbf{P}\left(t_{0}, n \tau\right)$, but a Markovian approximation to it in which the memory of the particle positions is lost after a time $\tau$. The Markovian approximation may be reasonable in some circumstances, and in fact it has been successfully used in geophysical flow problems. ${ }^{18,22,55}$ In this paper, we do not assume any Markovian hypothesis, and we work with the full matrix $\mathbf{P}\left(t_{0}, \tau\right)$ covering our time interval of interest and describing only the initial and final states of the transport process.

Despite not using any Markov assumption, replacing the continuous flow system by a discrete network introduces discretization errors. Even if the integration is done accurately, the initial and final locations of the transported particles are only specified up to a precision $\Delta$, given by the linear side of the boxes. This implies that our network approach does not display explicitly fluid structures smaller than the box length-scale $\Delta$.

\section{THE SURFACE FLOW NETWORK OF THE MEDITERRANEAN SEA}

We now apply the previous general procedures to build and analyze the flow network associated to a realistic surface flow in the Mediterranean sea.

The input velocity field originates from the Mediterranean Forecasting System Model (physics reanalysis component). It is a hydrodynamic model supplied by the Nucleus for European Modelling of the Ocean (NEMO), which solves primitive equations in spherical coordinates. NEMO has been implemented in the Mediterranean at an horizontal resolution of $1 / 16^{\circ}$, and 72 unevenly spaced vertical levels. ${ }^{56}$ It also slightly extends into the Atlantic in order to resolve the Strait of Gibraltar. The model uses vertical partial cells to fit the bottom depth shape. It is forced by momentum, water and heat fluxes interactively computed by bulk formulae using the $6 \mathrm{~h}, 1 / 4^{\circ}$ horizontal-resolution operational analysis and forecast fields from the European Centre for Medium-Range Weather Forecasts (ECMWF), while precipitation and river runoffs are provided by monthly mean datasets. The Dardanelles inflow is parameterized as a river and the climatological net inflow rates are considered. Assimilated data include sea level anomaly, remotely sensed sea surface temperature and in-situ temperature and salinity profiles.

We used daily horizontal velocity fields generated by the model in the whole Mediterranean basin during 10 years of simulation (2002-2011) selecting only one layer at a nominal depth of $7.9 \mathrm{~m}$. This layer extends in fact between 4.58 and $11.55 \mathrm{~m}$ depth so that it has a vertical extension of $6.97 \mathrm{~m}$. For the integration time scales used here (values of $\tau$ always below 3 months), we can reasonably neglect motion to other layers and consider only horizontal dynamics. ${ }^{11,39}$

\section{A. Discretization}

To switch from continuous space to discrete nodes, we partition the above-described horizontal near-surface Mediterranean layer into 3270 two-dimensional square boxes. We imposed the equal-area constraint defining the cells in a sinusoidal projection given by coordinates $x$ and $y$ related to the standard longitude $\varphi$ and latitude $\phi$ by

$$
x=\varphi \cos \phi ; \quad y=\phi .
$$

In these $x, y$ coordinates, boxes are squares of side $0.25^{\circ}$ or $\Delta=27.78 \mathrm{~km}$ (see Fig. 2). The area $\Delta^{2}$ of each box is $771.9 \mathrm{~km}^{2}$. The "amount of water in a box $B_{i}$ " is then related to its area $\Delta^{2}$ through a simple multiplication by the layer thickness $(6.97 \mathrm{~m})$, returning a value of $5.38 \times 10^{9} \mathrm{~m}^{3}$ per box.

The resolution of the model-generated velocity field is much finer than the discretization we use for network construction. In this sense, the dynamics represented in the flow network is a coarse-graining of the simulated Mediterranean flow, keeping the effect of the small scales only in a statistical sense. The most energetic features of the Mediterranean flow are mesoscale structures ${ }^{57}$ ranging from $10 \mathrm{~km}$ to a few hundred $\mathrm{km}$. With the value of $\Delta$ we use, our network 


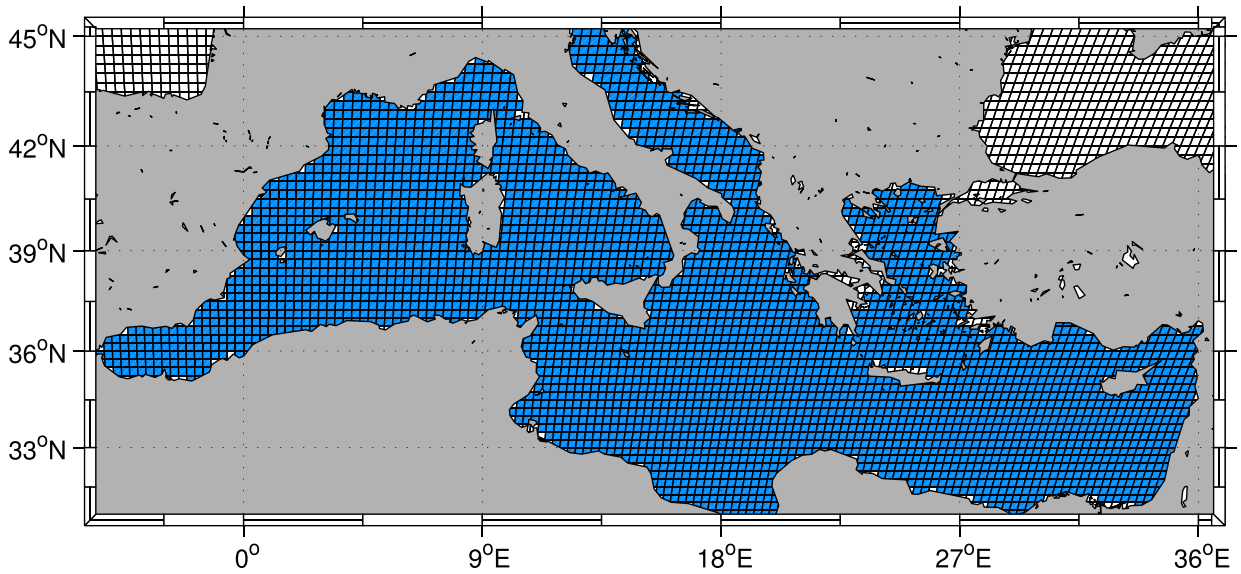

FIG. 2. Discretization of the Mediterranean sea (blue region) into $N=3270$ equal-area boxes $\left\{B_{i}, i=1\right.$, $\ldots, N\}$.

description displays most of the mesoscale range, and neglects submesoscales, which anyway are only marginally resolved by the NEMO implementation.

\section{B. Lagrangian simulation}

To characterize the transport phenomena, $N_{i}=500$ ideal fluid particles were released in each box $B_{i}$, providing enough statistics to estimate $\mathbf{P}\left(t_{0}, \tau\right)$. We simulated the motion of these $3270 \times 500=1.635 \times 10^{6}$ particles by integrating the trajectories in the velocity field using a fourthorder Runge-Kutta algorithm. The velocity at any arbitrary point in the sea is computed with a bilinear interpolation from the input data. We used a time step of 1 day (the same resolution as the data). We also tested shorter intervals using a cubic interpolation but no significant improvement was found. The two key-parameters of the simulations are the starting time $t_{0}$ and the tracking time $\tau$.

\section{Network construction}

The simulation provides the initial and final positions for each particle, allowing us to compute the transport matrix $\mathbf{P}\left(t_{0}, \tau\right)$ from Eq. (3). A directed link is established from node $i$ to node $j$ if and only if $\mathbf{P}\left(t_{0}, \tau\right)_{i j}$ is non-vanishing. In that case, its value gives the weight of such a link. Due to numerical limitations, some trajectories end up prematurely by "beaching" onto land areas outside of the partition $\left\{B_{i}\right\}$. Then, the denominator $N_{i}$ in Eq. (3) is taken as the number of particles still in the sea at the end of the integration time $\tau$. Since the beaching effect is small, affecting less than $5 \%$ of all particles in the longest simulations presented here (and only for near-shore boxes), we still assume in the following that the convenient equal-area condition remains approximately valid.

Note that the Lagrangian integration is done under the full resolution of the velocity field $\left(1 / 16^{\circ}\right)$. This means that particle trajectories contain the small-scale features produced by the model during time $\tau$. While such details are not explicitly present in the network description $\mathbf{P}\left(t_{0}, \tau\right)$ after coarse-graining the initial and final positions to the box size $\Delta$, their effects have been incorporated in a statistical way.

\section{NETWORK PROPERTIES}

We now interpret the transport matrices $\mathbf{P}\left(t_{0}, \tau\right)$, for several values of $t_{0}$ and $\tau$, as the adjacency matrices of directed and weighted flow networks. We can calculate for them all the standard quantities characterizing the topology of networks, such as degree, clustering, and betweenness. ${ }^{43}$ But following the aim stated in the Introduction, we will concentrate here in network quantities that can give insight in (horizontal) dispersion and mixing processes, and in the identification of coherent regions.

\section{A. Dispersion and mixing}

Important properties of geophysical flows depend on their dispersion characteristics, i.e., how far away can the fluid be transported during some time, and how diverse are the target regions. Mixing of fluid with different characteristics, another process of great geophysical relevance, will occur at a particular place if fluid from different origins arrives there at a particular time.

In dynamical systems approaches to flow processes, a standard way to quantify dispersion is by means of the finitetime Lyapunov exponent (FTLE). It is defined as ${ }^{12}$

$$
\lambda\left(\mathbf{x}_{0}, t_{0}, \tau\right)=\frac{1}{2|\tau|} \log \Lambda_{\max }
$$

where $\Lambda_{\max }$ is the maximum eigenvalue of the CauchyGreen strain tensor

$$
C\left(\mathbf{x}_{0}, t_{0}, \tau\right)=\left(\nabla \Phi_{t_{0}}^{\tau}\left(\mathbf{x}_{0}\right)\right)^{T} \nabla \Phi_{t_{0}}^{\tau}\left(\mathbf{x}_{0}\right),
$$

constructed from the Jacobian matrix $\nabla \Phi_{t_{0}}^{\tau}\left(\mathbf{x}_{0}\right)$ of the flow map. $M^{T}$ means the transpose of the matrix $M$. For $\tau>0$, this is the forward FTLE. By running the flow map backwards in time $(\tau<0)$, we get the backwards FTLE field, which quantifies the strength of mixing into a particular location. The interpretation of (5) is that an initial circle of infinitesimal diameter $\delta$ located at $\mathbf{x}_{0}$ at $t_{0}$ will become an ellipse of major axis $e^{\tau \lambda\left(\mathbf{x}_{0}, t_{0}, \tau\right)} \delta$ after being advected by the flow during a time $\tau$. The minor axis will be a decreasing function of $\tau$, contracting at an exponential rate related to a negative Lyapunov exponent that can be computed from the second eigenvalue of $C\left(\mathbf{x}_{0}, t_{0}, \tau\right)$. 
An obvious quantity in the network interpretation suitable to be related to dispersion and mixing is the degree of a node. Since our network is directed, we should distinguish between the in-degree $K_{I}(i)$, i.e., the number of links pointing to a particular node $i$, and the out-degree $K_{O}(i)$, the number of links pointing out of it. Figure 3 displays these quantities at the geographical locations defined by the nodes of the Mediterranean network for particular values of $t_{0}$ and $\tau$. High values of the degrees appear associated to the strong and unstable currents in the southern part of the basin. ${ }^{57}$ Low degree values are observed in regions where the circulation is rather slow, such as the Tunisian continental shelf and the semi-enclosed seas (e.g., Adriatic and Aegean). Generally, the values of the in- and out-degree tend to increase with $\tau$. With respect to the dependence on $t_{0}$, degree values tend to be slightly higher in winter than in summer.

A first problem in relating these network properties to the actual physics of dispersion and mixing is that their values are dependent on the spatial scales chosen for discretization (there is also a dependency on the numbers $N_{i}$ of particles used to compute the transport matrices, but it disappears for large $N_{i}$ ). This problem is easy to solve by recalling that every box has an associated area. Dealing first with the out-degree case for definiteness, $K_{O}(i)$ is proportional to the total area of all nodes that received some contents from the initial node $i$. This quantity has a well-defined meaning that can be related to the continuous flow dynamics with only a minor dependence on the discretization procedure. Since here all boxes have the same area $\Delta^{2}$, the area corresponding to the out-degree of node $i$ is $K_{O}(i) \Delta^{2}$. We can use generic ideas of chaotic dynamics to obtain heuristically a more precise relationship between two quantifiers of dispersion: the degree and the Lyapunov exponent. In regions dominated by hyperbolic structures, each of the fluid boxes will be stretched into a long and thin filament after a sufficiently long time $\tau$ (see Appendix A). If we want to compute the number of boxes reached by it, it is enough to consider its length, since the width quickly becomes smaller than the box size $\Delta$. Let us consider an initial line of length $L\left(t_{0}\right) \approx \Delta$ inside the initial box $B_{i}$. A small segment of it, of length $d l\left(t_{0}\right)$ at position $\mathbf{x}_{0} \in B_{i}$ will become elongated by a factor given by the local FTLE: $d l\left(t_{0}+\tau\right)=d l\left(t_{0}\right) e^{\tau \lambda\left(\mathbf{x}_{0}, t_{0}, \tau\right)}$. Integrating over the initial positions along the line, we get an estimation of the final length $L\left(t_{0}+\tau\right)$ of the filament. A better estimation $\bar{L}\left(t_{0}+\tau\right)$ of this length can be done by averaging over positions transverse to the line, to take into account different locations of the initial line in the box

$$
\bar{L}\left(t_{0}+\tau\right) \approx \frac{1}{\Delta} \int_{B_{i}} d \mathbf{x}_{0} e^{\tau \lambda\left(\mathbf{x}_{0}, t_{0}, \tau\right)},
$$

0

5

10

15

20
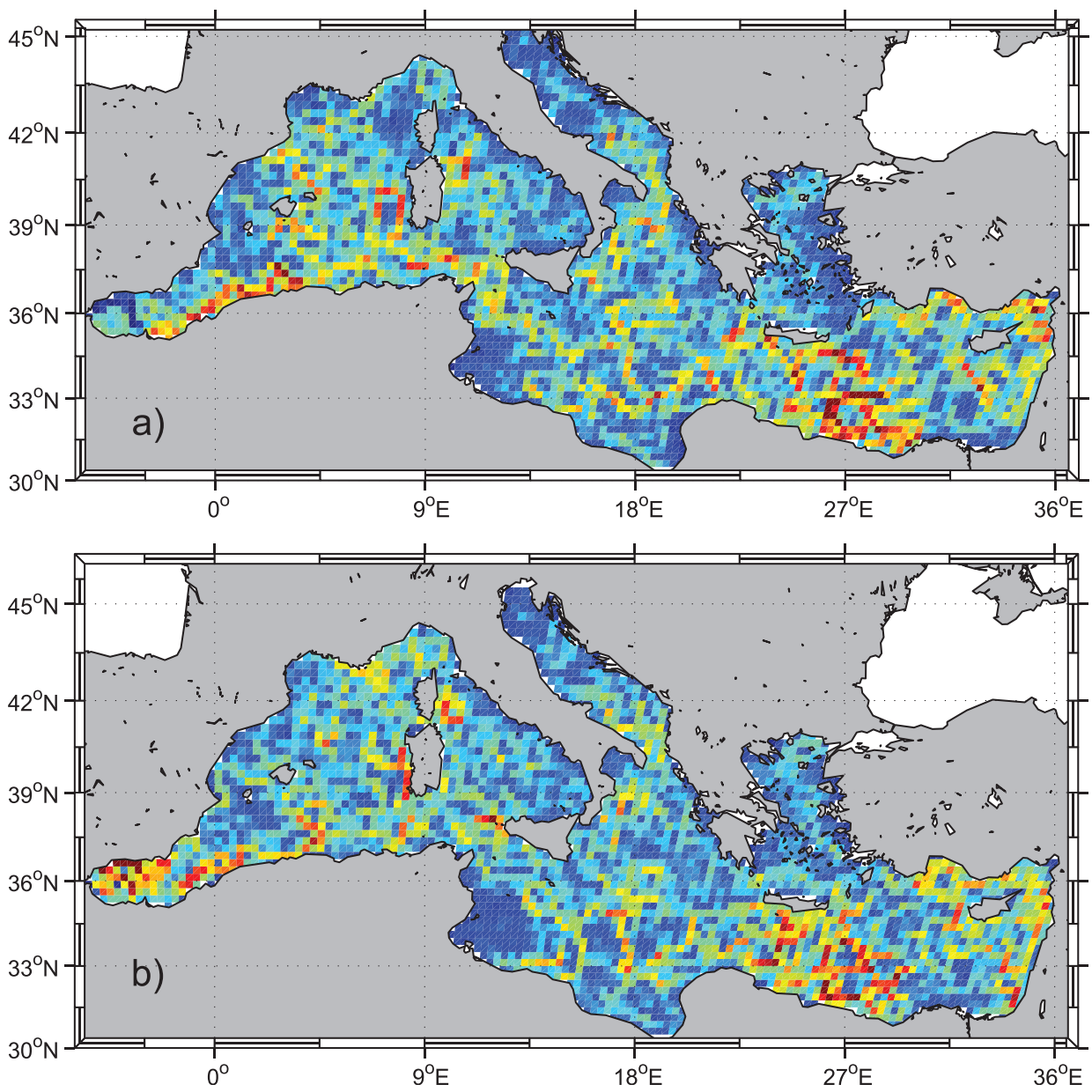

FIG. 3. Degree of the nodes in the flow network defined by $\mathbf{P}\left(t_{0}, \tau\right)$, for $t_{0}=$ July 1 st 2011 and $\tau=15$ days. (a) The in-degree $K_{I}(i)$. (b) The out-degree $K_{O}(i)$. 
where the longitudinal and transverse integrations have been combined into the integration of $\mathbf{x}_{0}$ over the area $B_{i}$. The area of the boxes covered by the filament is $\mathcal{A}\left(t_{0}+\tau\right) \approx$ $\bar{L}\left(t_{0}+\tau\right) \Delta$ so that the out-degree of the initial box will be

$$
K_{O}(i)=\frac{\mathcal{A}\left(t_{0}+\tau\right)}{\Delta^{2}} \approx \frac{1}{\Delta^{2}} \int_{B_{i}} d \mathbf{x}_{0} e^{\tau \lambda\left(\mathbf{x}_{0}, t_{0}, \tau\right)} \equiv\left\langle e^{\tau \lambda\left(\mathbf{x}_{0}, t_{0}, \tau\right)}\right\rangle_{B_{i}} .
$$

Thus, we have a useful relationship between a natural quantity in the network description of fluid flows and a standard characterization of dispersion in the dynamical systems approach to such flows: the degree of a node associated to a box is the average or coarse-graining of the stretching factor $e^{\tau \lambda}$ in that box. We can check the validity of the above heuristic arguments by comparing directly the values of $K_{O}(i)$ obtained from our flow network and the right-hand-side of (8). Figure 4 shows an example of FTLE field obtained at time $t_{0}=$ July 1 st 2011 and $\tau=15$ days. Figure 5 shows the clear correlation between the two quantities. Three values of $\tau$ are plotted to appreciate the general validity of the relationship. We attribute the deviations with respect to the exact identity to the fact that the filament-type arguments are only valid for sufficiently large $\tau$ and in regions dominated by strain. Also, our arguments neglect the presence of filament foldings that sometimes would occupy the same box, and of associated saturation effects. In addition quantization, effects arising from the discrete nature of $K_{O}$ are visible at small degree values.

Expression (8) suggests defining

$$
H_{i}^{0}\left(t_{0}, \tau\right) \equiv \frac{1}{\tau} \log K_{O}(i),
$$

so that

$$
\left\langle e^{\tau \lambda\left(\mathbf{x}_{0}, t_{0}, \tau\right)}\right\rangle_{B_{i}}=e^{\tau H_{i}^{0}\left(t_{0}, \tau\right)} .
$$

From the convexity of the exponential function, we have $H_{i}^{0}\left(t_{0}, \tau\right) \geq\left\langle\lambda\left(\mathbf{x}_{0}, t_{0}, \tau\right)\right\rangle_{B_{i}}$. The previous expressions are reminiscent of the properties of the topological entropy of a dynamical system, as giving the exponential growth in time of the length of a material line. ${ }^{58}$ Pushing forward the analogy, we can define a sequence of Rényi-like entropies ${ }^{59}$ associated to a particular node $i$

$$
H_{i}^{q}\left(t_{0}, \tau\right) \equiv \frac{1}{(1-q)|\tau|} \log \sum_{j=1}^{N}\left(\mathbf{P}\left(t_{0}, \tau\right)_{i j}\right)^{q},
$$

which we call network entropies. Due to their dependence on the finite-size of the partition, they are related to the $\epsilon$-entropies discussed by Boffetta et al. ${ }^{60}$ Note, however, that here the transport matrix involves only two states of the trajectories, separated by an interval of time $\tau$ which remains finite, and the dependence on the initial location, box $B_{i}$, is kept. The entropies $H_{i}^{0}$ and $H_{i}^{1}$ should be understood as defined by the limits $q \rightarrow 0$ and $q \rightarrow 1$, respectively. All the network entropies measure the diversity in the amounts of fluid received by the nodes connected to a given box, but weighting them in different ways: In $H_{i}^{0}$, all nodes are counted equally independently of the amount of water they receive so that it informs only about the degree as seen in Eq. (9); for increasing values of $q$ nodes receiving more water are weighted with increasing strength. Although the network entropies have been introduced here in the particular context of flow networks, we note that they can be defined for any weighted network, giving generalizations of the degree to quantify the unevenness of the weight distribution towards the nodes connected to a given one.

Applying l'Hôpital's rule to the definition of the network entropy of order $q=1$, one gets

$$
H_{i}^{1}\left(t_{0}, \tau\right)=-\frac{1}{\tau} \sum_{j=1}^{N} \mathbf{P}\left(t_{0}, \tau\right)_{i j} \log \mathbf{P}\left(t_{0}, \tau\right)_{i j} .
$$

It gives the amount of information (per unit of time) gained by observing the position of a particle at time $t_{0}+\tau$, knowing that it was initially (time $t_{0}$ ) somewhere in box $B_{i}$. This quantity is precisely the discrete finite-time entropy studied by Froyland and Padberg-Gehle. ${ }^{29}$ Figure 6 shows its spatial
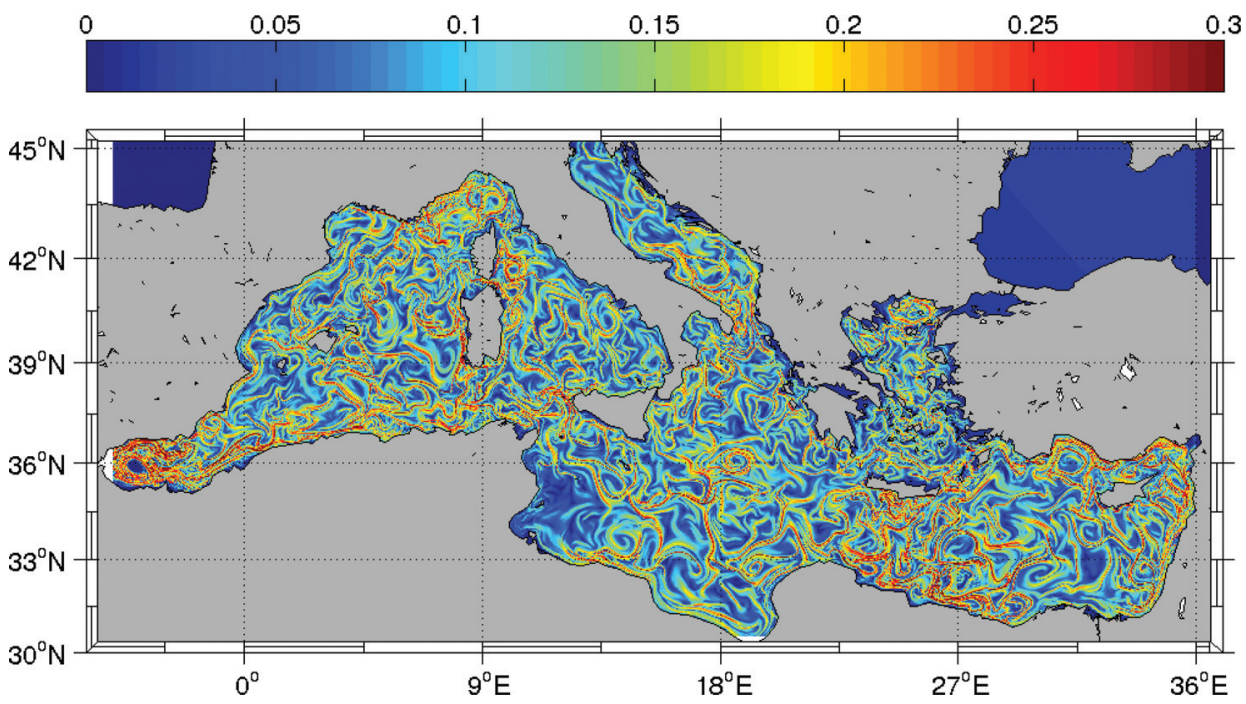

FIG. 4. An example of forward FTLE field $\lambda\left(\mathbf{x}_{0}, t_{0}, \tau\right)$ at $t_{0}=$ July 1 st 2011 and $\tau=15$ days. Color bar in day ${ }^{-1}$. 


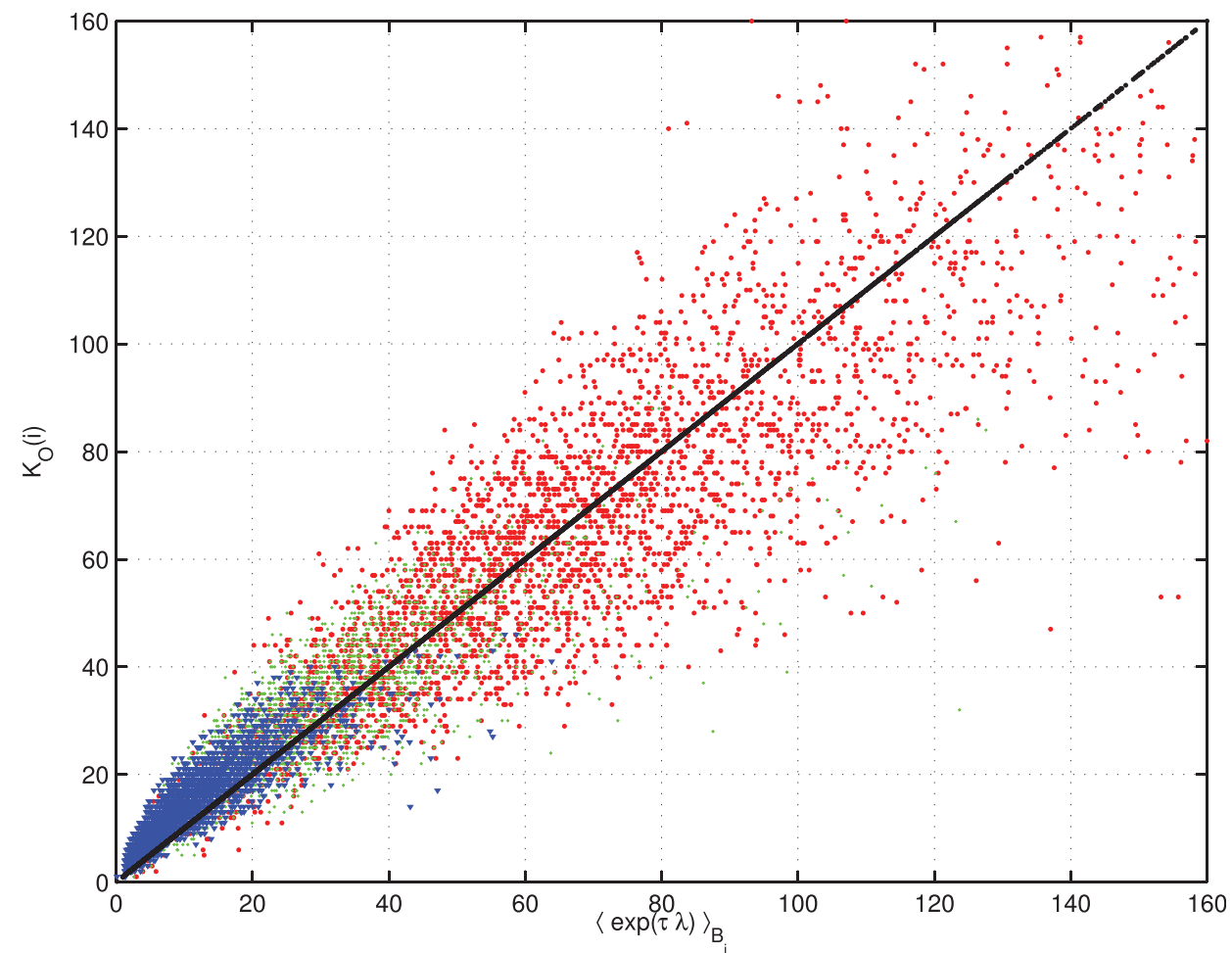

FIG. 5. Values of the out-degree $K_{O}(i)$ of each node $i$ vs the average value of the stretching factor $e^{\tau \lambda}$ in that node. $t_{0}=$ July 1 st 2011. Blue symbols are from $\tau=15$ days, green from $\tau=30$ days, and red from $\tau=60$ days. Black line is the main diagonal. distribution in the Mediterranean sea for particular values of $t_{0}$ and $\tau$.

The standard Pesin-like results relating the metric or Kolmogorov-Sinai entropy to the sum of positive Lyapunov exponents $^{60-62}$ suggest that, at least for large $\tau$, the entropy $H_{i}^{1}$ would give a good approximation to the values of the FTLE field averaged over each box $B_{i}: \lambda_{i}\left(t_{0}, \tau\right)$ $\equiv\left\langle\lambda\left(\mathbf{x}_{0}, t_{0}, \tau\right)\right\rangle_{B_{i}} \approx H_{i}^{1}\left(t_{0}, \tau\right)$. Appendix A gives calculations supporting this claim in an heuristic way. Figure 7 shows the geographical distribution of $\lambda_{i}\left(t_{0}, \tau\right)$, and Fig. 8 compares both quantities for several values of $\tau$. The entropies tend to be slightly larger than the Lyapunov exponents for $\tau=15$ days, but both quantities approach each other and become well correlated for larger $\tau$.

For definiteness, we have been discussing quantities related to the forward time evolution: out-degree, forward
Lyapunov exponents, etc. The network entropies can also be defined for the backward time evolution. Construction of the backwards-dynamics network can be achieved by redoing the launching of particles and running the Lagrangian integration for negative time, or much simpler, by recognizing ${ }^{29}$ that the backward evolution is given by the matrix

$$
\mathbf{P}\left(t_{0}+\tau,-\tau\right)_{i j}=\frac{\mathbf{P}\left(t_{0}, \tau\right)_{j i}}{\sum_{k=1}^{N} \mathbf{P}\left(t_{0}, \tau\right)_{j k}} .
$$

The network entropies in Eq. (11) can now be directly computed for the backward flow network defined by $\mathbf{P}\left(t_{0}+\tau,-\tau\right)$, and they will be related to backwards Lyapunov fields, which give a measure of mixing of fluid coming from different origins. As an example, we show in Fig. 9 the relationship between the backwards entropy $H_{i}^{1}\left(t_{0}+\tau,-\tau\right)$ and the coarse-grained
0

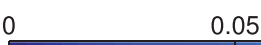
0.05 0.1

0.15
0.25

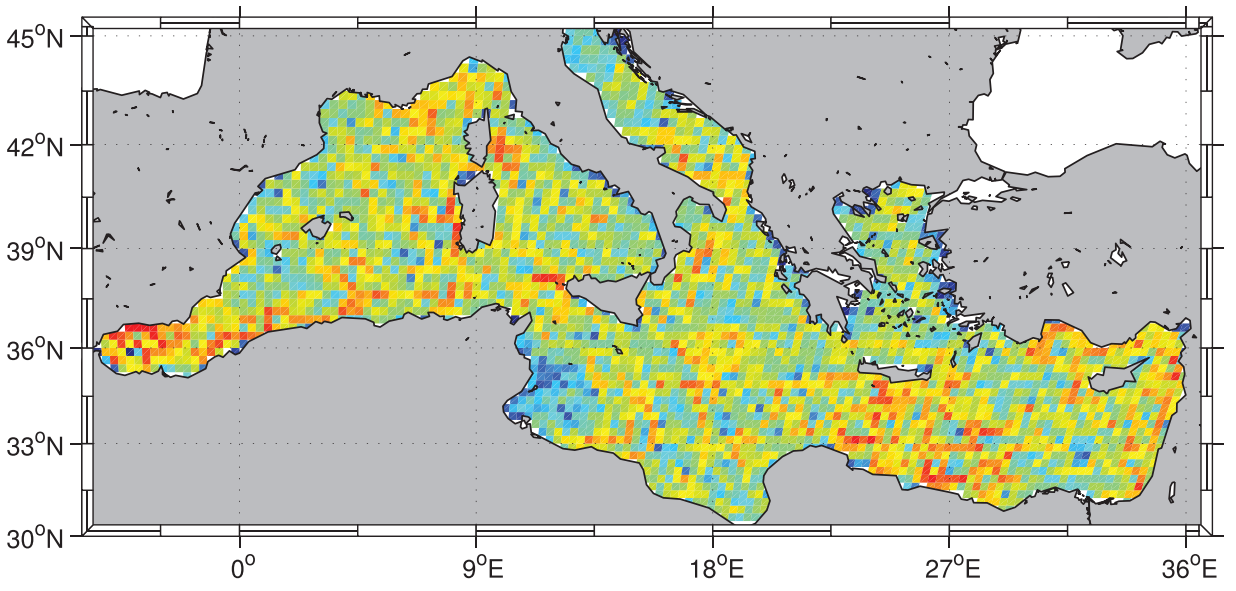

FIG. 6. The network entropy $H_{i}^{1}\left(t_{0}, \tau\right)$, for $t_{0}=$ July 1 st 2011 and $\tau=15$ days. Color bar in day ${ }^{-1}$. 

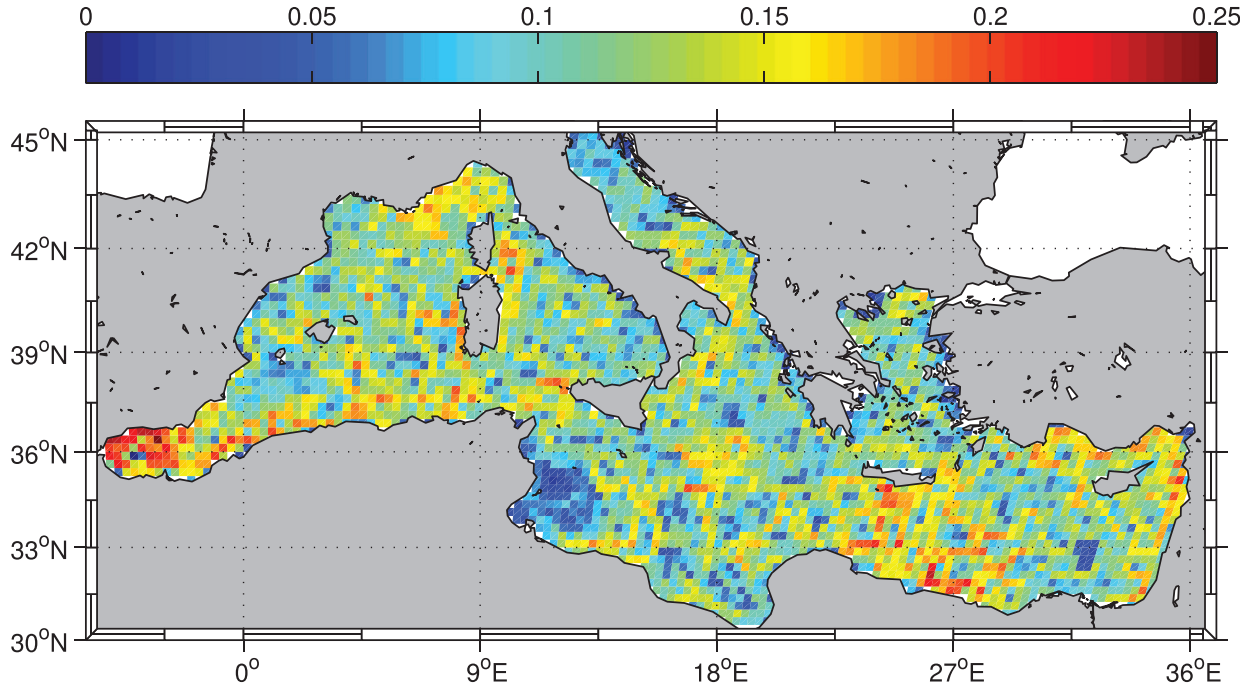

FIG. 7. Coarse-graining of the Lyapunov field in Fig. 4 into the discretization boxes: $\lambda_{i}\left(t_{0}, \tau\right) \equiv\left\langle\lambda\left(\mathbf{x}_{0}, t_{0}, \tau\right)\right\rangle_{B_{i}} . \quad t_{0}$ $=$ July 1 st 2011 and $\tau=15$. Color bar in day ${ }^{-1}$. backwards Lyapunov exponent $\lambda_{i}\left(t_{0}+\tau,-\tau\right)$. Again both quantities are similar for sufficiently large $\tau$ and the same qualitative features as in Fig. 8 are observed.

Summarizing this section, we have defined a family of entropy-like quantities completely in terms of the transport matrix characterization of the flow network. At least two of them, $H_{i}^{0}$ and $H_{i}^{1}$ are related to standard dispersion and mixing quantifiers in the description of fluid flows. The higher order entropies $H_{i}^{q}$ are related to the generalized Lyapunov exponents ${ }^{60,62}$ characterizing successive moments of the Lyapunov field, as discussed in Appendix A. We do not claim that these relationships are exact for finite values of $\tau$ and $\Delta$. Instead, we find numerical deviations from them (Figs. 5, 8, and 9) which decrease for increasing $\tau$. We expect the same to happen when decreasing $\Delta$. The important point is that, once the network matrix $\mathbf{P}\left(t_{0}, \tau\right)$ has been constructed, the entropies in Eq. (11) provide a computationally very cheap way to assess quantities of geophysical interest such as local dispersion, stretching, and mixing. In fact, the simplest network quantifiers such as the in- and out-degrees are already suitable for that, being related to $H_{i}^{0}$. The qualitative information displayed in Figs. 3(b) and 6 or 7 is very similar. Also, even if we should have $H_{i}^{0} \geq H_{i}^{1} \approx \lambda_{i}$, in our examples the numerical values of $H_{i}^{0}$ are only slightly larger than those of $H_{i}^{1}$. We have to mention that we have been working under the hypothesis of boxes $\left\{B_{i}\right\}$ of equal areas. Expression (11) would need corrections in a more general case. See, for example, the case of $H_{i}^{1}$ in Froyland and Padberg-Gehle. ${ }^{29}$

\section{B. Identification of coherent regions}

\section{Coherent regions as network communities}

Most work in the dynamical systems approach to fluid transport aims at identifying "barriers to transport" locating

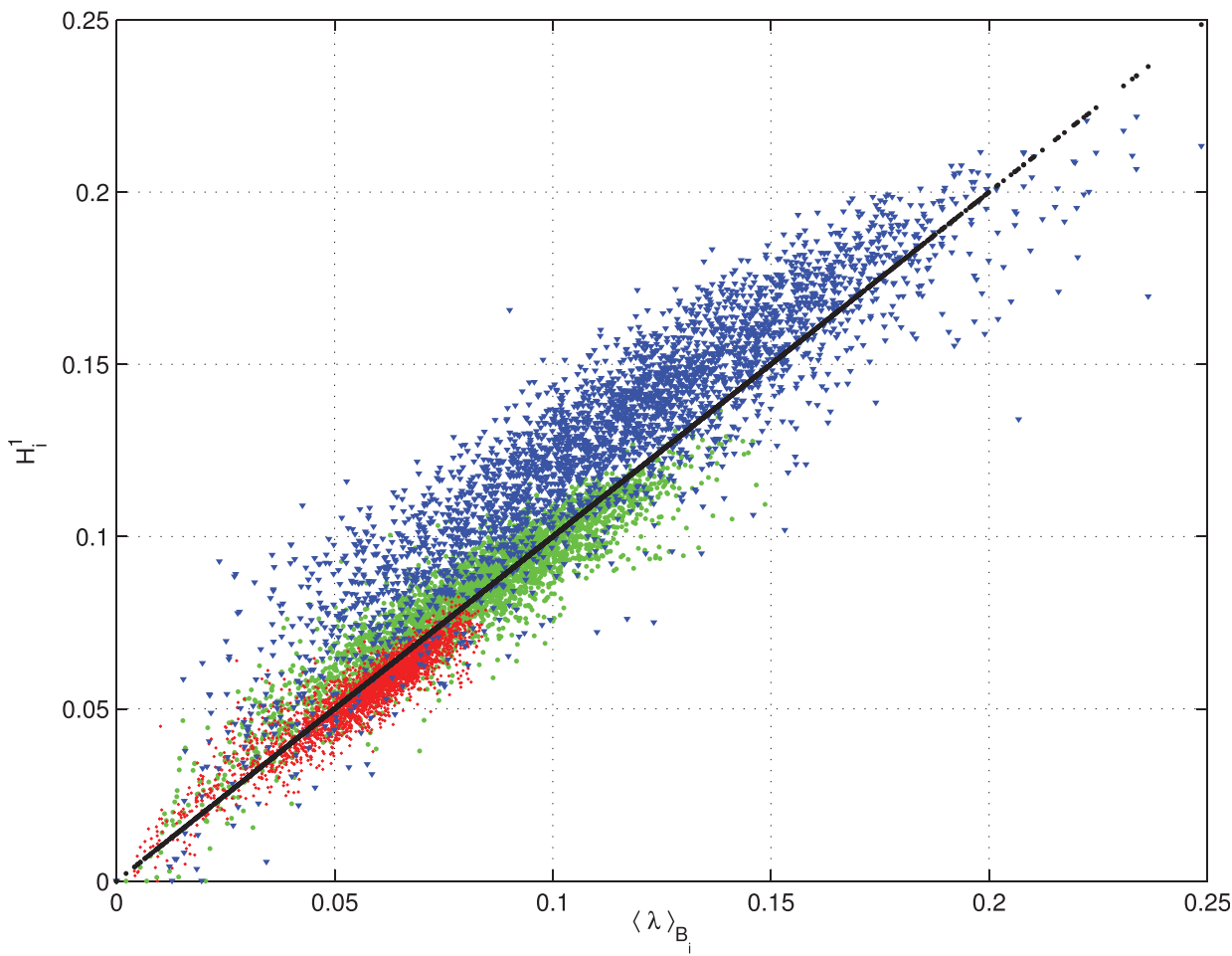

FIG. 8. Values of the network entropy $H_{i}^{1}\left(t_{0}, \tau\right)$ of each node $i$ vs the average value of the Lyapunov exponent in that node, $\lambda_{i}\left(t_{0}, \tau\right) . t_{0}=$ July 1 st 2011 . Blue symbols are from $\tau=15$ days, green from $\tau=30$ days, and red from $\tau=60$ days. Black line is the main diagonal. 


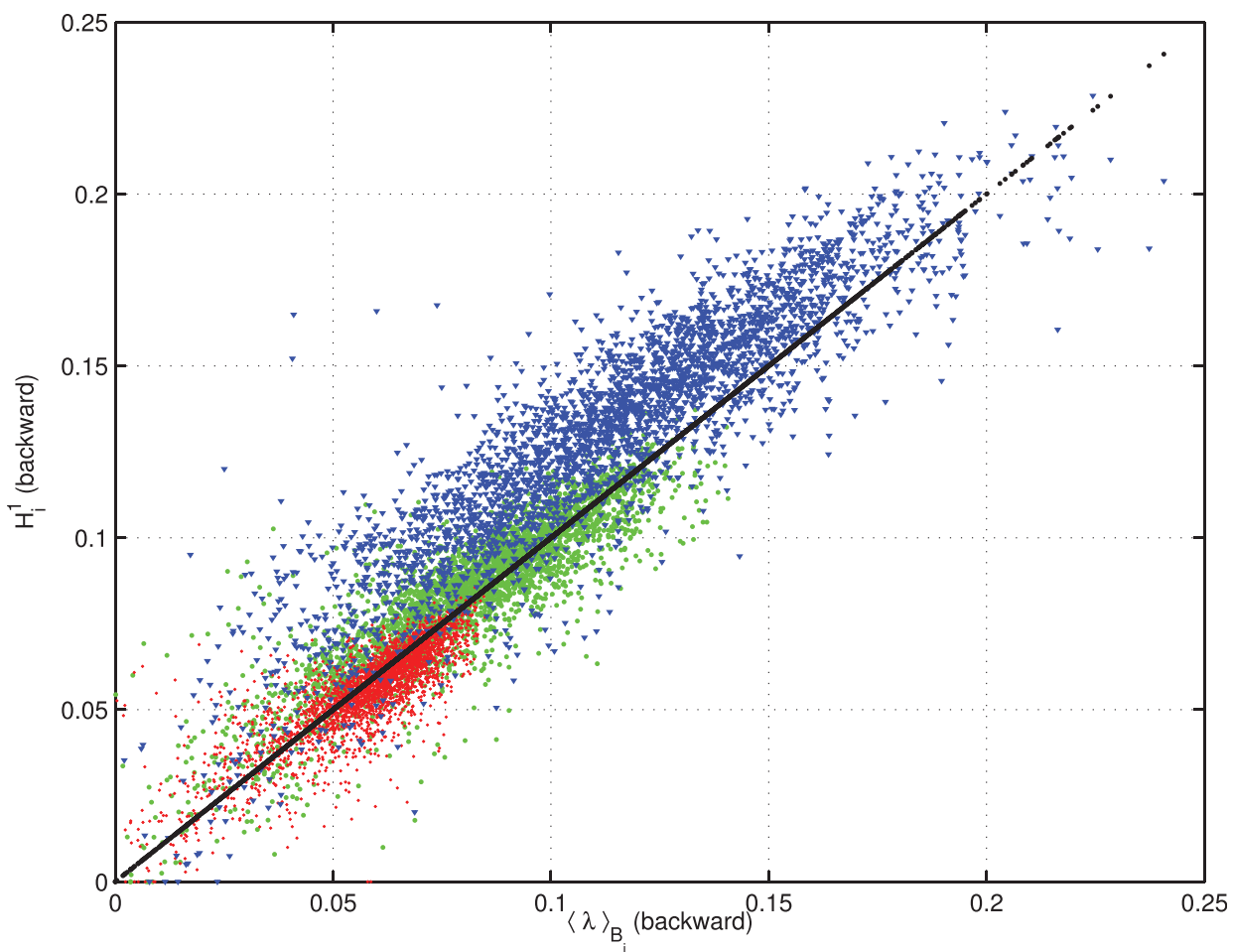

FIG. 9. Values of the network entropy $H_{i}^{1}$ at each node $i$, computed from the backwards-dynamics network given by $\mathbf{P}\left(t_{0}+\tau,-\tau\right)$ (Eq. (13)), vs the average value of the backwards Lyapunov exponent in that node, $\lambda_{i}\left(t_{0}+\tau,-\tau\right)$. $t_{0}=$ July 1 st 2011. Blue symbols are from $\tau=15$ days, green from $\tau=30$ days, and red from $\tau=60$ days. Black line is the main diagonal. the borders of regions that do not exchange much fluid among them. The set-oriented approach focusses on the regions themselves rather than on the borders. Almostinvariant fluid regions have been defined as regions of the fluid domain remaining relatively isolated (according to a suitably defined metrics) from the rest of the fluid. ${ }^{15,16}$ In generic time-dependent flows, these regions will not be fixed in space but they will be transported by the mean flow, and the concept of coherent pairs, relating initial and final set positions has been developed. ${ }^{19,20,22}$ Formulating this problem in the context of network theory would require building on techniques for bipartite graphs. In our present case study, the global flow in the Mediterranean sea, land masses play an important role in restricting the flow so that coherent regions that remain fixed with respect to the coasts are the most relevant ones for many applications. In particular, when considering environmental conservation strategies and marine reserves, ${ }^{36,38,39}$ one looks for the connectivity among marine zones, or provinces, ${ }^{39}$ occupying localized regions of the sea. Thus, we focus here on finding a partition of the sea into self-coherent, or almost-invariant regions, associated to relatively stable circulation patterns, from the point of view of network theory. We want these regions to be well-mixed internally, and with little interchange with the exterior. In the language of networks, this translates to partitioning the network into subgraphs with high internal connectivity, and small connectivity among them. This is the standard problem of community detection in networks, ${ }^{43,63-66}$ for which many different and powerful techniques are available. In fact, most of the approaches used so far to partition fluid motion into almost-invariant sets ${ }^{15,16,37}$ employ classical spectral techniques for graph partition, ${ }^{43}$ which use the eigenvectors or singular vectors of the transport matrix (or other matrices derived from it). We note that the methodologies in Refs. 15 and 16 find almost-invariant sets in the sense that loss and gain of fluid is minimized. But the condition of strong internal mixing, which we consider important in geophysical applications, is not imposed.

Here, we address the community detection problem with a state-of-art network-theory approach, the Infomap algorithm. ${ }^{67}$ The method is based on the probability flow of random walks in the network moving with transition probabilities given by the adjacency matrix $\mathbf{P}\left(t_{0}, \tau\right)$, and on exploiting the properties of information compression in the description of that probability flow. Infomap finds the partition of the network minimizing the average size of the codeword needed to describe inter- and intracommunity transitions. A succinct description of the method is provided in Appendix B. We believe this methodology is specially suited to partition flow networks for the following reasons: First, it takes into account the "direction" and "weight" of each link, important characteristics defining our flow network. The standard spectral methods and most modularityoptimization algorithms take as input a symmetrized version of the network. Second, Infomap does not require to fix a priory the number of communities forming the domain partition. Third, Infomap does not impose similar sizes to the communities so that it does not suffer from the "resolution limit" 68 restricting the minimum community size detectable by most algorithms, including spectral methods. This is important in geophysical flow networks since ocean structures of different sizes coexist in the sea, some of them arising from geographical accidents, bathymetry, etc.

The method has also some limitations. One of them is the "field of view limit" sition matrix $\mathbf{P}\left(t_{0}, \tau\right)$. In general, this imposes that the detected communities are only those with intense intracommunity connections (clique-like). For our application, this 
feature may become convenient since Infomap will identify as communities only regions well mixed internally by the flow.

Since Infomap consider random walkers exploring the network with the transition probabilities in the matrix $\mathbf{P}\left(t_{0}, \tau\right)$, one is tempted to confuse these walkers with the Lagrangian particles advected by the flow. But this is not correct. $\mathbf{P}\left(t_{0}, \tau\right)$ contains relationships between initial and final positions of particles after a time $\tau$, but does not describe in detail the trajectories at intermediate times. In addition, it can not be used beyond that time since in time-dependent velocity fields flow connectivity will change with the initial time $t_{0}$, defining the dynamic network. Infomap unveils the graph structures present in the single matrix $\mathbf{P}\left(t_{0}, \tau\right)$ by releasing random walkers that evolve in a virtual time not directly related to the physical time.

Hydrodynamical provinces delimited by Infomap in the Mediterranean surface flow were already studied by Rossi et al. ${ }^{39}$ who discussed also their implications for the design of marine reserves. Here, we concentrate in the technical aspects and compare with alternative methods.

\section{Quality parameters}

A standard way to asses the quality of a network partition is by computing a modularity parameter. ${ }^{43,70}$ But this involves comparison with a random null model than in the case of flow networks has no obvious meaning. Then we prefer to use alternative quantifiers with a direct interpretation in terms of fluid connectivity. Here, we define a coherence ratio and a mixing parameter.

If coherent regions $A$ are understood as almost-invariant areas of fluid, this means that they are mapped by the flow nearly into themselves after a time $\tau$

$$
\Phi_{t_{0}}^{\tau}(A) \approx A .
$$

To measure how well this is achieved, one can introduce the coherence ratio $^{15,16}$

$$
\rho_{t_{0}}^{\tau}(A)=\frac{m\left(A \cap \Phi_{t_{0}+\tau}^{-\tau}(A)\right)}{m(A)},
$$

where as before, $m(C)$ is the area of set $C$, but it can be generalized to other measures. We have $\rho_{t_{0}}^{\tau}(A) \leq 1$ and values close to unity indicate that $A$ is a truly almost-invariant set.

In our discrete set-up, we consider sets $A$ made of our boxes $\left\{B_{i}, i=1, \ldots, N\right\}: A=\cup_{i \in \mathcal{I}} B_{i}$, where $\mathcal{I}$ is the set of indices identifying the boxes $B_{i}$ making $A$. The coherence ratio is now ${ }^{15,16}$

$$
\rho_{t_{0}}^{\tau}(A)=\frac{\sum_{i, j \in \mathcal{I}} m\left(B_{i}\right) \mathbf{P}\left(t_{0}, \tau\right)_{i j}}{\sum_{i \in \mathcal{I}} m\left(B_{i}\right)}
$$

For a partition of the fluid domain into $p$ communities or provinces: $\mathcal{P}=\left\{A_{1}, \ldots, A_{p}\right\}$, a global quality figure of the partition is

$$
\rho_{t_{0}}^{\tau}(\mathcal{P}) \equiv \frac{1}{p} \sum_{k=1}^{p} \rho_{t_{0}}^{\tau}\left(A_{k}\right)
$$

where again a good partition would be indicated by a value close to 1 . When communities are of very different sizes, it may be appropriate to weight the average in Eq. (17) with these sizes, but we keep the present definition to allow comparison with previous works.

Physically, we can say, that $\rho_{t_{0}}^{\tau}(\mathcal{P})$ represents the fraction of tracers that at time $t_{0}+\tau$ are found in the same province where they were released at time $t_{0}$. The definition involves the initial and final positions, but gives no information on the particle trajectories in between. Note that coherence ratios measure fluid exchanges between provinces, but do not quantify how strong the internal mixing is.

The second quantifier we use is a mixing parameter devised to assess how strongly the flow mixes fluid inside communities. To define the mixing parameter $M_{t_{0}}^{\tau}(A)$ inside a set $A$, we first define a transport matrix conditioned to represent just the transport occurring inside $A$ (more precisely, transport by trajectories that start and end in $A$ )

$$
\mathbf{R}\left(t_{0}, \tau \mid A\right)_{i j}=\frac{\mathbf{P}\left(t_{0}, \tau\right)_{i j}}{\sum_{k \in \mathcal{I}} \mathbf{P}\left(t_{0}, \tau\right)_{i k}}, i, j \in \mathcal{I} .
$$

As before, $\mathcal{I}$ is the set of indices identifying the boxes $B_{i}$ making $A$. The mixing parameter is a normalized version of the sum inside $A$ of the entropies associated to the transition probabilities in $\mathbf{R}\left(t_{0}, \tau \mid A\right)$

$$
M_{t_{0}}^{\tau}(A)=\frac{-\sum_{i, j \in \mathcal{I}} \mathbf{R}\left(t_{0}, \tau \mid A\right)_{i j} \log \mathbf{R}\left(t_{0}, \tau \mid A\right)_{i j}}{Q_{A} \log Q_{A}} .
$$

$Q_{A}$ is the number of boxes in $A$. The maximum value, $M_{t_{0}}^{\tau}(A)=1$, is reached when fluid is dispersed from each box in $A$ to all the others uniformly $\left(\mathbf{R}_{i j}=1 / Q_{A}, \forall i, j \in \mathcal{I}\right)$. A global quantification of the internal mixing in a community partition $\mathcal{P}=\left\{A_{1}, \ldots, A_{p}\right\}$ is given by

$$
M_{t_{0}}^{\tau}(\mathcal{P})=\frac{\sum_{k=1}^{p} m\left(A_{k}\right) M_{t_{0}}^{\tau}\left(A_{k}\right)}{\sum_{k=1}^{p} m\left(A_{k}\right)} .
$$

Here, we have weighted the different communities according to their size.

\section{Communities in the Mediterranean surface flow}

The outputs of the Infomap algorithm applied to the flow network defined by $\mathbf{P}\left(t_{0}, \tau\right)$, for increasing values of $\tau$, are shown in Fig. 10. Each community $A_{k}$ is colored with the value of its coherence ratio $\rho_{t_{0}}^{\tau}\left(A_{k}\right)$. We see that most coherence values are rather high. The global mixing parameter has only moderate values (see caption of Fig. 10), but it increases with $\tau$. The main coast-constrained regions appear clearly outlined (the Tyrrhenian, the Adriatic, and the Aegean), but also other areas defined only by persistent circulation patterns (the three-gyre system in the Adriatic and the Balearic front). We refer to Rossi et al. ${ }^{39}$ for a thorough interpretation of the hydrodynamic provinces in relation with surface 


0.9
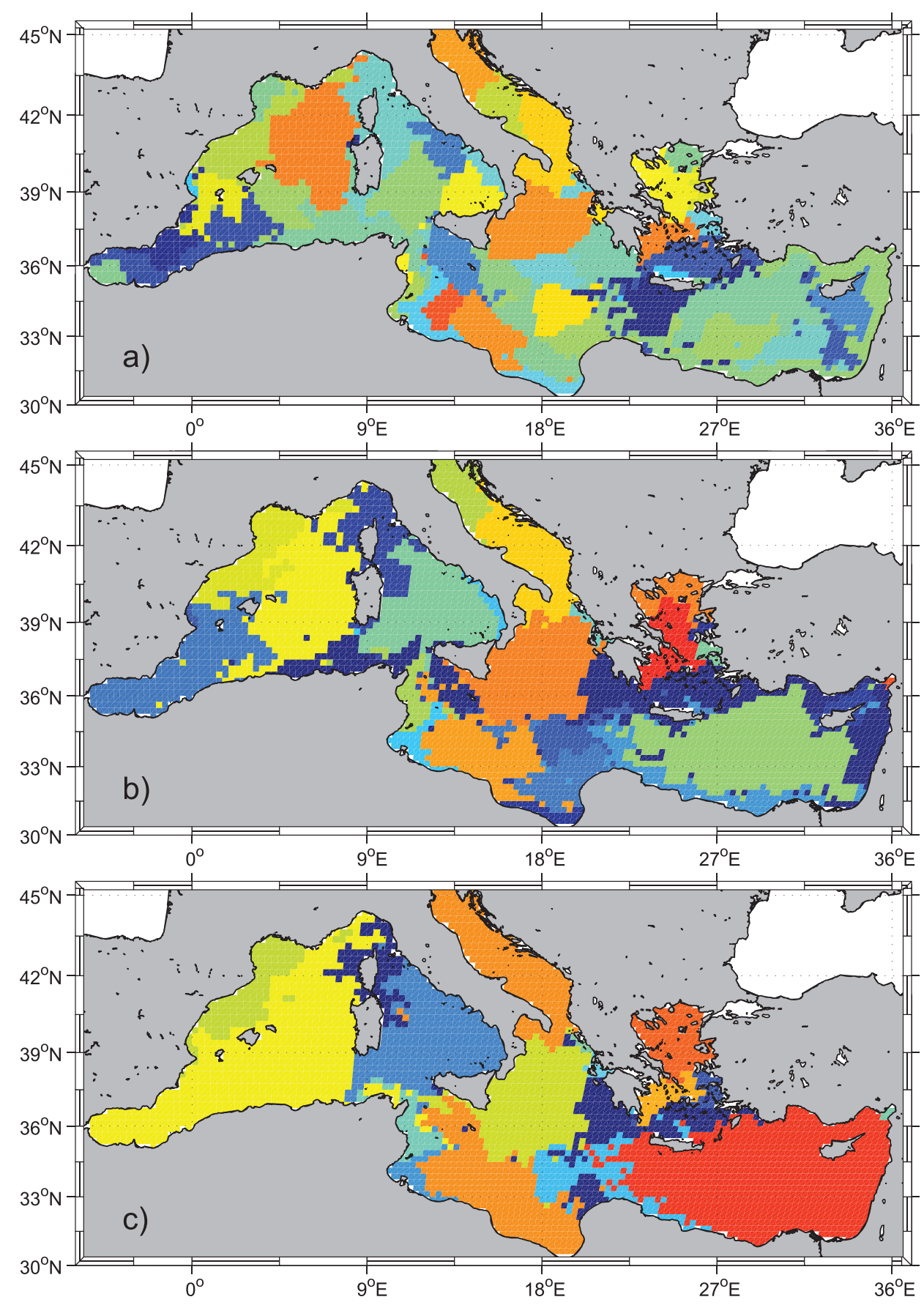

FIG. 10. Infomap partition of flow networks in the Mediterranean sea, defined by $\mathbf{P}\left(t_{0}, \tau\right)$, into communities or provinces for increasing values of $\tau$. Each province is colored by its coherence ratio value from Eq. (16), as given in the color bar. In all panels, $t_{0}=$ July 1 st 2011 . (a) $\tau=30$ days; the number of communities is $p=56$, the global coherence $\rho_{t_{0}}^{\tau}(\mathcal{P})=0.76$, and the global mixing $M_{t_{0}}^{\tau}(\mathcal{P})=0.47$. (b) $\tau=60$ days; $p=33$, $\rho_{t_{0}}^{\tau}(\mathcal{P})=0.73$, and $M_{t_{0}}^{\tau}(\mathcal{P})=0.54$. (c) $\tau=90 \quad$ days; $\quad p=22, \quad \rho_{t_{0}}^{\tau}(\mathcal{P})$ $=0.80$, and $M_{t_{0}}^{\tau}(\mathcal{P})=0.59$. circulation patterns and known eco-regionalization of the Mediterranean basin. Note that there is no obvious relationship between the size of a community and its coherence. Both large and small provinces may have indeed moderate $(<0.6)$ or high $(>0.8)$ coherence ratios. The detection of small communities confirms that Infomap is not affected by the "resolution limit." 68

Communities merge and in average become larger with increasing $\tau$ so that their number decreases. Fig. 11(a) shows the growth of the mean area as a function of $\tau$ for the same case $t_{0}=$ July 1 st 2011 shown in Fig. 10. The standard deviation of the area distribution is also displayed as error bars. It shows a significant dispersion in the area of the communities identified, especially for larger $\tau$, revealing properly the multiscale character of oceanic transport processes. For small $\tau$, community areas seem mainly controlled by the time of integration (there is no sufficient time for the flow to manifest highly inhomogeneous dispersion) but only marginally determined by the intrinsic properties of the flow. As commented above, detecting communities of widely different sizes is a great capability of Infomap, whereas other methodologies constrain the communities to be of similar sizes. The inset Fig. 11(b) shows how the number of communities decreases when $\tau$ increases. 
a)

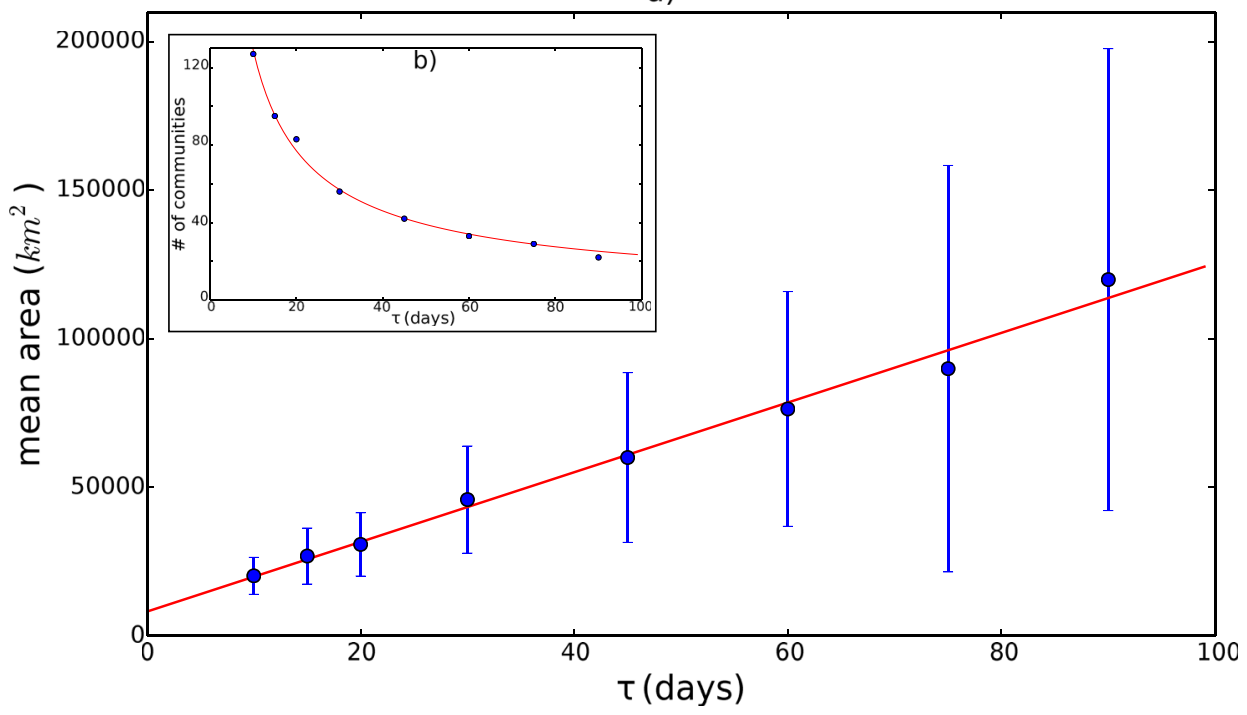

FIG. 11. Panel (a) shows the mean area (dots) of the communities detected by the Infomap algorithm for $t_{0}=$ July 1 st 2011 as a function of $\tau$. The straight line is a fit to the diffusive-growth-like relationship Area $=8109.6+1173.8 \times \tau$. The error bars indicate the standard deviation of the area distribution. Note the large dispersion in community sizes. The upper left inset (b) shows the decay of the number of communities with $\tau$.

\section{Average descriptions}

Because of the turbulent nature of oceanic motions, the community decomposition changes with $t_{0}$. Some communities (even of small size) are repeatedly observed while some others appear and disappear when changing $t_{0}$. In order to identify persistent communities, i.e., those whose limits are relatively stable in space and time, we explore two averaging procedures leading to a mean-"climatological"-community partition. In a first approach, we average a number of matrices $\mathbf{P}\left(t_{0}, \tau\right)$ corresponding to the same starting date (e.g., January 1st) for the ten different years of the data set (e.g., January 1st 2002, January 1st 2003, etc., until January 1st 2011). Figure 12 shows the Infomap partition of the

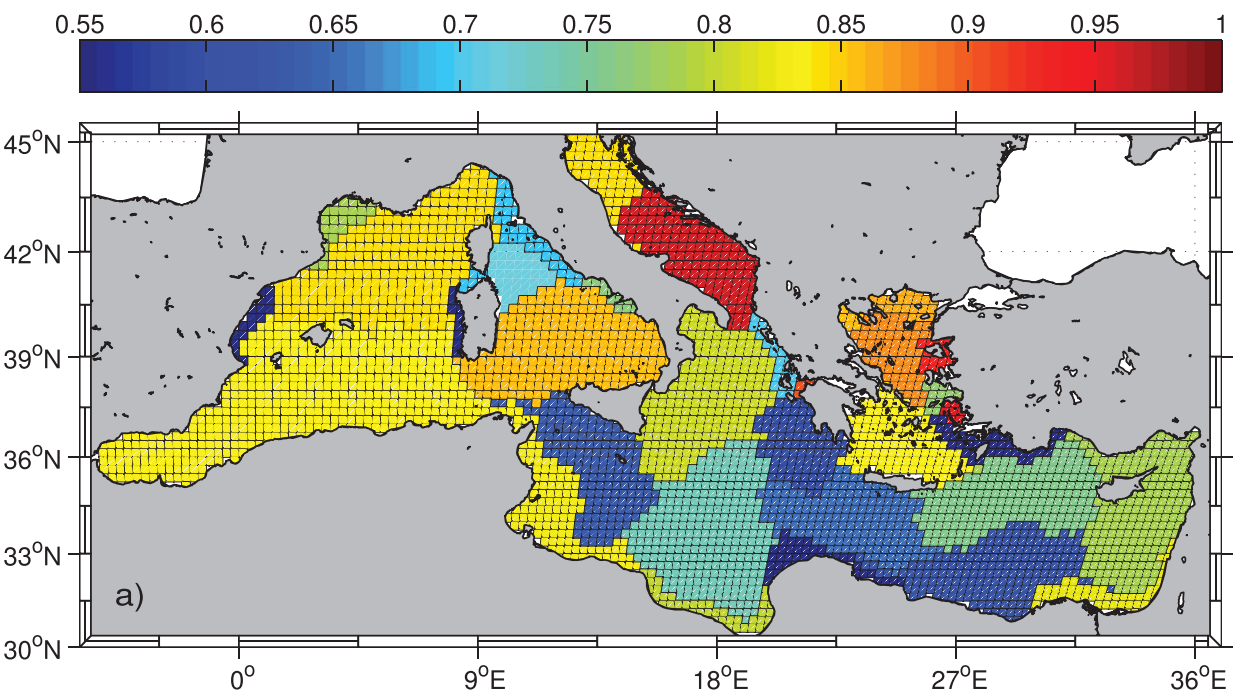

FIG. 12. Infomap communities obtained from the average networks given by $\overline{\mathbf{P}\left(t_{0}, \tau\right)}$, with $\tau=30$ days. Each community is colored by its coherence ratio. (a) The average is over the 10 matrices corresponding to $t_{0}=$ January 1 st in 10 years (2002-2011) of simulation; the number of communities is $p=34$, the global coherence $\rho_{t_{0}}^{\tau}(\mathcal{P})=0.78$, and the global mixing $M_{t_{0}}^{\tau}(\mathcal{P})=0.68$. (b) The average is over the 10 matrices corresponding to $t_{0}=$ July 1 st in the 10 years 2002-2011; $\quad p=30, \quad \rho_{t_{0}}^{\tau}(\mathcal{P})$ $=0.77$, and $M_{t_{0}}^{\tau}(\mathcal{P})=0.69$.

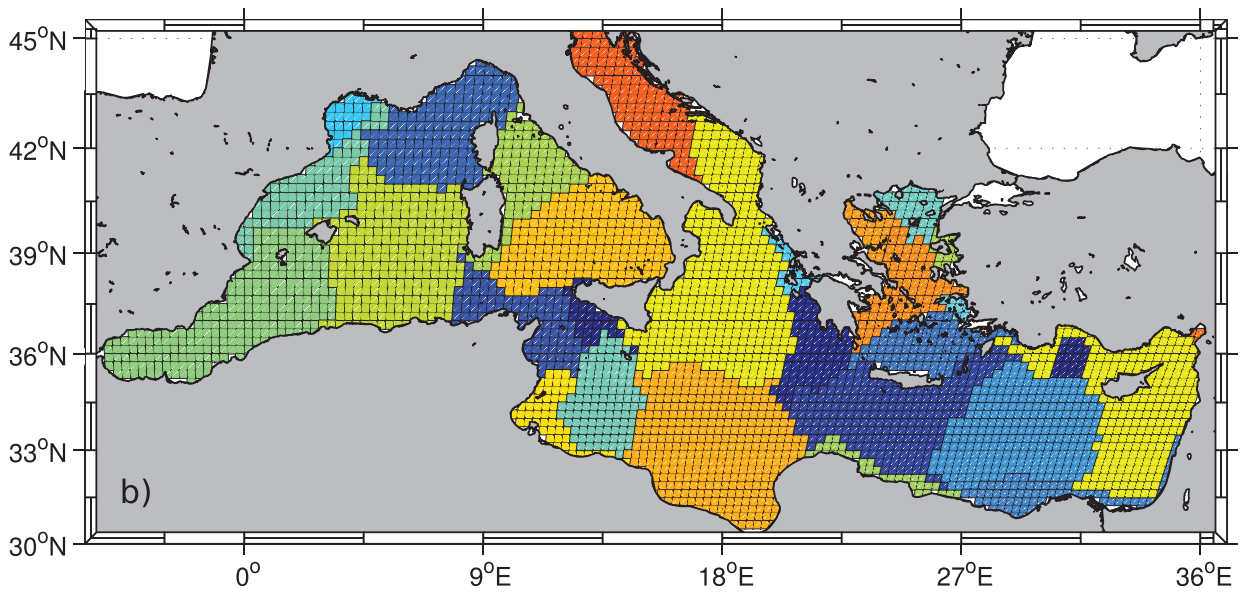


network defined by the average matrix $\overline{\mathbf{P}\left(t_{0}, \tau\right)}$ made with the ten matrices $\mathbf{P}\left(t_{0}, \tau\right)$ using the same starting date for each of the 10 years (2002-2011). An example of $t_{0}$ in winter and another one in summer are displayed. The figure shows the most persistent communities for a particular month, averaging out the variability occurring over ten years. We remark than some communities have a rather small size (most of them reflecting shallow oceanic regions such as continental shelves), and that there is some inter-seasonal variability.

A second approach to obtain average or climatological description of the community partition is illustrated in Fig.
13. Instead of applying only once Infomap on an averaged transport matrix, it is here applied 10 times separately on the 10 transport matrices corresponding to the same starting date for each of the 10 years (2002-2011). The color at a particular location of Fig. 13 indicates the frequency of occurrence (in these 10 partitions) at that location of a border between communities. Then, greener color indicates a more persistent community border. The strongest lines would represent true "barriers to transport" which remain fixed in space. Fuzzier lines may indicate intermittent border appearance, but also a larger wandering amplitude. Figures 13(a) and 13(b) display
0

0.1

0.2

0.3

0.4

0.5

0.6

0.7

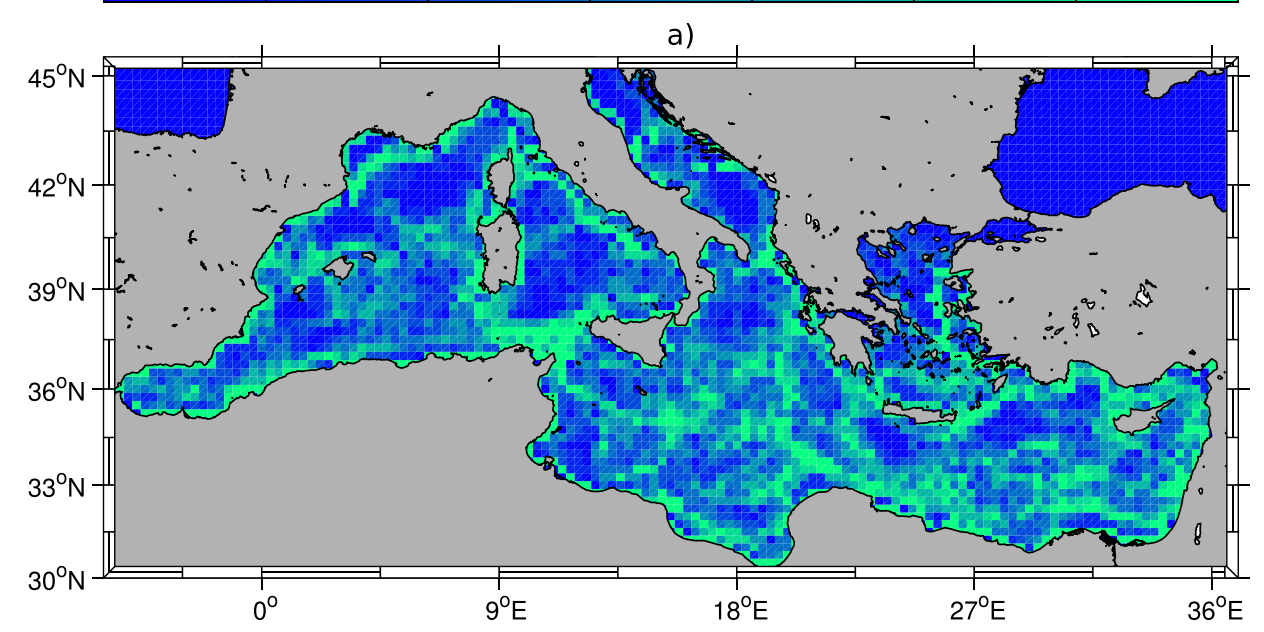

b)

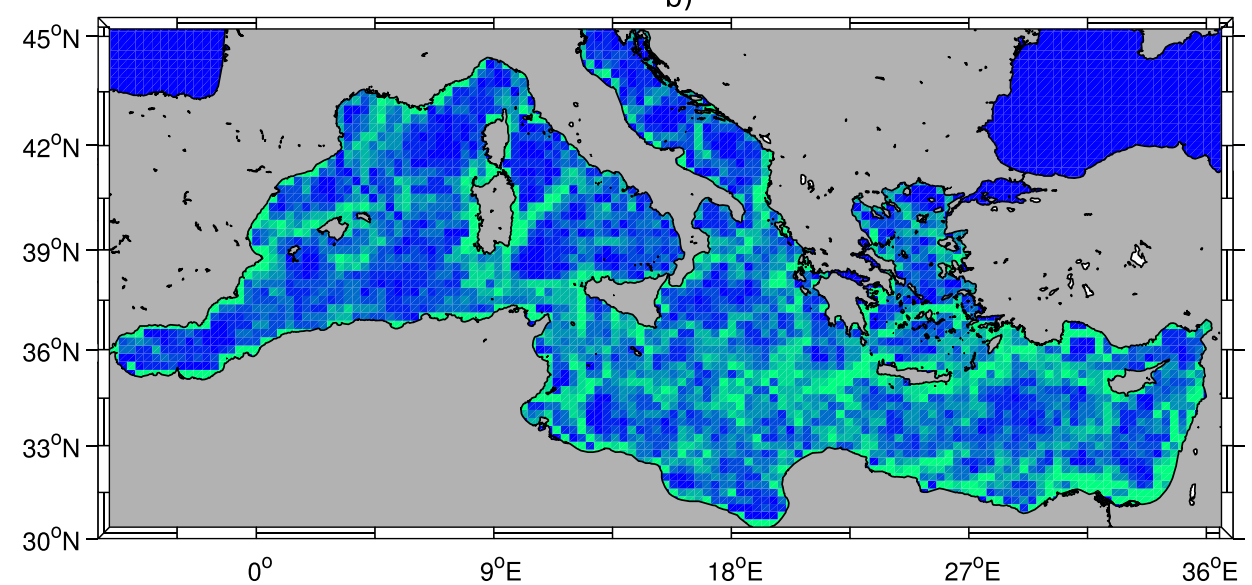

c)

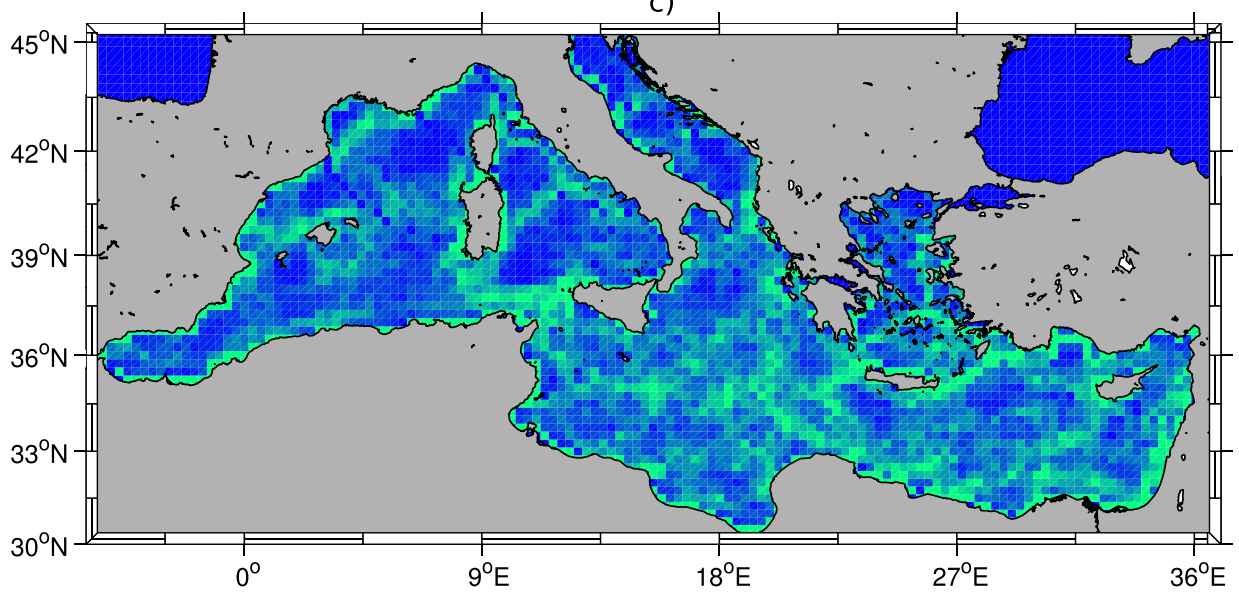

FIG. 13. Persistence of community borders over time: Color code indicates the proportion of times one of the borders between communities has appeared at a given location. $\tau=30$ days. (a) $t_{0}=$ January $1 \mathrm{st}$ of (2002-2011). (b) $t_{0}=$ July $1 \mathrm{st}$ of (2002-2011). (c) The average of the two previous panels, eliminating the seasonal information. 
the situation in the same winter and summer days as in Fig. 12. Figure 13(c) shows a combination of them, equivalent to showing the barrier persistence sampled twice a year during the ten years.

\section{Comparison with spectral partitioning}

Different methods based on the spectral properties of transport matrices have been previously used to identify and locate almost-invariant sets in flows. ${ }^{15-18,37}$ They exploit the fact that for a set to remain almost invariant after the effect of the flow, it has to be related with eigenvectors of $\mathbf{P}\left(t_{0}, \tau\right)$ with eigenvalues close to 1 . Here, we compare our partitioning obtained by Infomap with the one from those spectral methods. To be specific, we consider the method described by Froyland and Dellnitz. ${ }^{15}$ The technique in this last paper obtains a partition $\mathcal{P}$ minimizing in an approximate way the global coherence $\rho_{t_{0}}^{\tau}(\mathcal{P})$. To this end, it computes eigenvectors associated to nearly vanishing eigenvalues of the Laplacian matrix ${ }^{43}$ obtained from the symmetric part of $\mathbf{P}\left(t_{0}, \tau\right)$ and combines them using a fuzzy c-means clustering algorithm. ${ }^{15}$ Note that this approach eliminates any directionality information present in the transport network. Also, the c-means clustering can define as single community pieces of the ocean which are geographically disjoint or in fact quite far apart, if this enhances the coherence defined in Eq. (17). In the method, one has to specify the number of eigenvectors being combined (we choose it to be 10) and the number of communities in the partition. Figure 14 shows the results using the same average matrix $\overline{\mathbf{P}\left(t_{0}, \tau\right)}$ as in Fig. 12(b), and imposing a partition in 10 and in 14 communities. The change in the number of communities leads to rearrangements in the Tyrrhenian, the central Mediterranean, the Aegean, and the Levantine basin. In panel (a), some of the communities are made of disjoint pieces. Larger number of communities decreases the global coherence ratio (see caption of Fig. 14). If we try to increase the number of communities approaching the one given by Infomap, we find that the clustering algorithm becomes unstable. Instabilities also occur when the number of links in the transport network becomes too high (as occurring, for example, when increasing $\tau$ beyond 1 month).

When compared with the Infomap decomposition, we see that several of the boundaries coincide. But there are important differences, such as the wider range of community sizes and the sharper details revealed by Infomap. This is because a constraint of similar sizes for the communities associated to the same eigenvector needs to be imposed in the spectral method. When clustering several eigenvectors together, this limitation is partially bypassed

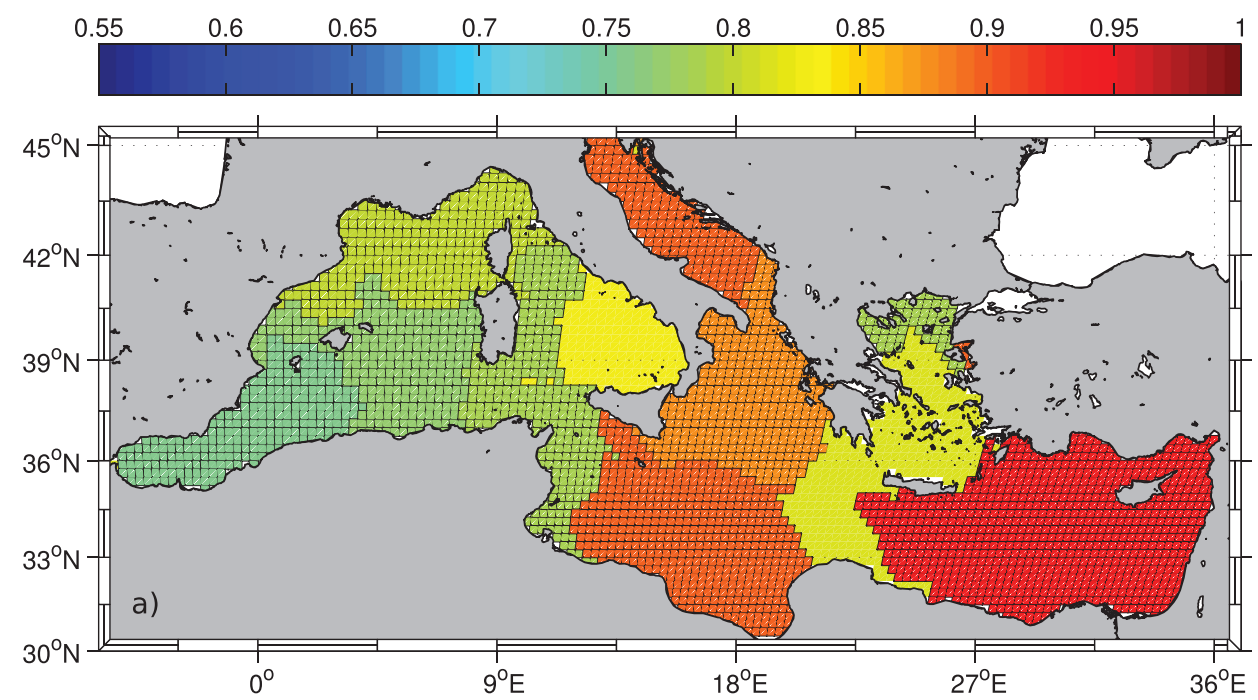

FIG. 14. Community decomposition by the spectral method with fuzzy cmeans clustering described in Froyland and Dellnitz. ${ }^{15}$ The matrix used is the same average $\overline{\mathbf{P}\left(t_{0}, \tau\right)}$ as in Fig. 12(b), i.e., with $t_{0}=$ July $1 \mathrm{st}$, averaged in the ten years 2002-2011, and $\tau=30$ days. Ten eigenvalues are used. (a) The number of communities is fixed to be $p=10$; the global coherence is $\rho_{t_{0}}^{\tau}(\mathcal{P})=0.85$, and the global mixing is $M_{t_{0}}^{\tau}(\mathcal{P})=0.62$. In the Aegean, the southern yellow community is the only independent one: the portions of the Aegean further north are clustered by the c-means algorithm as being part of the same province as areas in the central Mediterranean with the same color. (b) $p=14 ; \rho_{t_{0}}^{\tau}(\mathcal{P})=0.78$, $M_{t_{0}}^{\tau}(\mathcal{P})=0.64$.

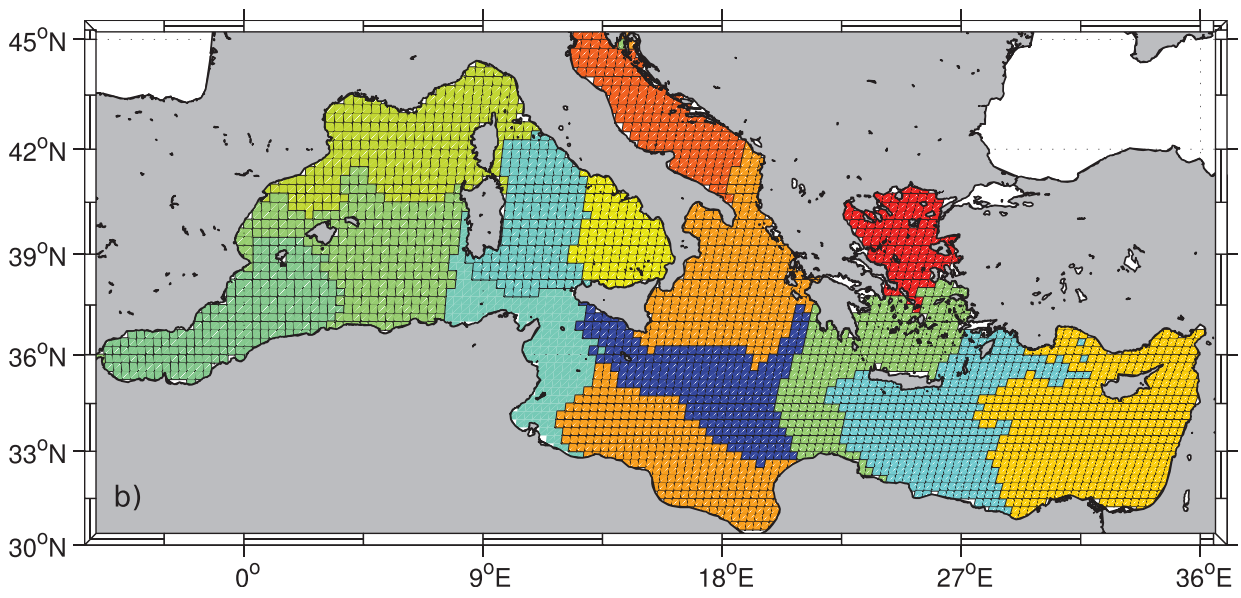


but still not removed. The values of the coherence ratio are of the same order or somehow larger for the spectral method, but note that the number of spectral communities has been kept much smaller to avoid the instabilities in the clustering algorithm. Since merging two communities into a single one increases the global coherence, joining some of the Infomap communities in Fig. 12 until arriving to 10 or 14 communities as in Fig. 14 would give rather large values of $\rho_{t_{0}}^{\tau}(\mathcal{P})$. As expected, the global mixing parameter is larger for the Infomap partition, but only by a small amount, reflecting that, even if internal mixing is not imposed in the spectral method, it is achieved to a reasonable extent.

From the methodological point of view, Infomap presents the advantage of determining itself the number of communities in the partition, whereas this needs to be fixed a priori (as well as the number of eigenvectors to be clustered) in the spectral approach. On the other hand, the spectral method is formulated as an algorithm to minimize the global coherence ratio, a quantity with a clear physical meaning. The quantity optimized by Infomap is a codeword length given in Eq. (B1) of Appendix B, an abstract information-theoretic object without a clear physical meaning. The heuristic interpretation of the optimization process leads to the "large internal-small external connectivity" property for the communities, but a more rigorous understanding of the Infomap procedure is clearly needed. ${ }^{67,69}$

The results of this section indicate that the Infomap methodology proposed here to identify coherent fluid regions seems more appropriate than spectral methods when a wide range of community sizes is expected, when internal mixing is a key parameter, or to minimize user input (such as entering the number of communities). Spectral methods seem appropriate when one is looking precisely for the sets defined mathematically as almost-invariant, the coherence ratio describes well the desired properties of the partition, and one expects a limited range of sizes.

\section{CONCLUSIONS}

We have used the concept of flow networks to obtain a discretized view of transport processes in geophysical contexts. Once the fluid motion is cast into the graph-theory framework, powerful techniques from this field become available to investigate the fluid transport processes. In this paper, we have improved, using network concepts, the characterization of geophysical dispersion and mixing process, as well as the identification of coherent fluid regions. One of the simplest network descriptors, the degree of a node, gives direct information on local stretching properties, classically associated to the finite-time Lyapunov exponents and their distributions. Thus, the out-degree at a particular node is quantitatively related to the fluid stretching at that location in the time-forward direction and quantifies fluid dispersion. The in-degree is related to backwards stretching and thus to the mixing of fluid from different origins.
A family of network-entropy functions has been defined, aiming at describing higher order statistical properties of fluid stretching (and then of dispersion and mixing) in terms of the network adjacency matrix. One of them, $H_{i}^{0}$ is simply the logarithm of the degree. Another one, $H_{i}^{1}$, is the discrete finite-time entropy studied by Froyland and PadbergGehle. ${ }^{29}$ We find numerically that it provides a good estimation of the coarse-grained finite-time Lyapunov exponent. We expect higher order entropies to be related to the generalized Lyapunov exponents ${ }^{60,62}$ that characterize successive moments of the Lyapunov field. Further work is needed to assess the validity of these properties more rigorously, beyond the heuristic and numeric arguments given in Sec. IV A and in Appendix A.

This paper considered flow networks in the geophysical context, but it is anticipated that the concepts are equally valid in more general fluid dynamics context, and even apply to more abstract flows in the phase space of dynamical systems. ${ }^{15,34,35}$ Also, the network entropies defined here can be used to characterize the local properties of general weighted networks beyond the degree and the node strength.

As a second application in which the network representation provides useful insights, we have investigated the identification of coherent regions in the ocean flow, ${ }^{36,38,39}$ regions that are similar to almost invariant sets ${ }^{15,16}$ but for which the presence of strong internal mixing is also desired. We find in the network-theory toolbox a useful community detection technique, Infomap, that takes into account the directed and weighted nature of the flow network, and that finds partitions of the geophysical flow with the required characteristics without restricting the range of community sizes. We have argued that these characteristics make it an interesting alternative to spectral methods to identify the desired coherent regions, although we also recognize that a substantial clarification of the physical meaning of the minimization process involved in Infomap is needed. This partition of the sea into coherent provinces has already been used to evaluate larval connectivity and to inform the design of marine protected areas. ${ }^{39}$ The present implementation of the method deals only with regions fixed with respect to geographic boundaries. Tools from the study of bipartite networks would be needed to find moving coherent regions such as vortices. ${ }^{20,22}$

We believe that the representation of fluid motion as a transport or flow network, allowing the use of powerful techniques from graph theory, will continue to provide novel insights into the nonlinear processes occurring in our planet, most of them related to fluid transport.

\section{ACKNOWLEDGMENTS}

We acknowledge financial support from FEDER and MINECO (Spain) through the ESCOLA (CTM2012-39025C02-01) and INTENSE@COSYP (FIS2012-30634) projects, and from European Commission Marie-Curie ITN program (FP7-320 PEOPLE-2011-ITN) through the LINC project (No. 289447). The simulated velocity field used here was generated by MyOcean (http://www.myocean.eu/). The authors thank two anonymous reviewers for their constructive comments. 


\section{APPENDIX A: RELATIONSHIP BETWEEN NETWORK ENTROPIES AND STRETCHING STATISTICS}

In this Appendix, we derive heuristically relationships between the network entropies defined in Sec. IV A and Lyapunov exponent statistics (in the two-dimensional case). Fig. 15 illustrates the basic ideas. The assumptions are that dynamics is mainly hyperbolic in the region of interest, and that $\tau$ and the size $\Delta$ of the fluid boxes $\left\{B_{i}, i=\right.$ $1, \ldots, N\}$ are such than the image of the boxes by the flow after a time $\tau$ are thin and long filaments. Boxes in the partition have been roughly aligned with expanding and contracting directions to make easier the heuristic arguments.

The point is to estimate the values of the matrix elements $\mathbf{P}\left(t_{0}, \tau\right)_{i k}$ given in Eq. (2)

$$
\mathbf{P}\left(t_{0}, \tau\right)_{i k}=\frac{m\left(B_{i} \cap \Phi_{t_{0}+\tau}^{-\tau}\left(B_{k}\right)\right)}{m\left(B_{i}\right)} .
$$

The quantity in the numerator of Eq. (A1) is the area of the doubly dashed thin filament in the left of Fig. 15. If we assume that the forward FTLE $\lambda\left(\mathbf{x}_{0}, t_{0}, \tau\right)$ is approximately constant for $\mathbf{x}_{0}$ in this region, we have $m\left(B_{i} \cap \Phi_{t_{0}+\tau}^{-\tau}\left(B_{k}\right)\right) \approx$ $\Delta^{2} \exp \left(-\lambda_{i k} \tau\right)$ (see Fig. 15), where $\lambda_{i k}$ is this constant value. In consequence, $\mathbf{P}\left(t_{0}, \tau\right)_{i k} \approx \exp \left(-\lambda_{i k} \tau\right)$ if $B_{k}$ is one of the boxes containing part of the image $\Phi_{t_{0}}^{\tau}\left(B_{i}\right)$ of $B_{i}$, and $\mathbf{P}\left(t_{0}, \tau\right)_{i k}=0$ elsewhere.

Spatial features in typical forward FTLE fields are thin filaments with nearly constant value $\lambda$. They are elongated along the expanding directions ${ }^{9,12}$ and have widths of the order of $l \exp (-\lambda \tau)$, where $l$ is the size of the velocity field inhomogeneities, i.e., the size of the Eulerian structures driving the flow. Then, the uniformity condition we are imposing is $\Delta<l$, i.e., discretization boxes smaller than Eulerian structures. In our Mediterranean example, $\Delta$ is smaller than the dominant mesoscale structures in the sea, but some of the smaller features in the velocity field can have some impact on the validity of the uniformity condition.

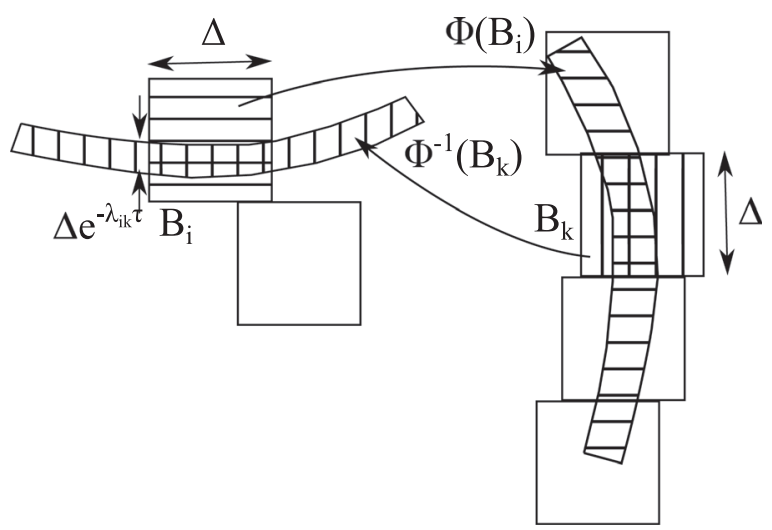

FIG. 15. Schematics of the stretching (forward and backwards in time) of fluid boxes of sidelength $\Delta$ corresponding to network nodes. $\Phi\left(B_{i}\right)$ is a shortcut for $\Phi_{t_{0}}^{\tau}\left(B_{i}\right)$, and $\Phi^{-1}\left(B_{k}\right)$ is a shortcut for $\Phi_{t_{0}+\tau}^{-\tau}\left(B_{k}\right)$. $\lambda_{i k}$ is the value of the forward FTLE $\lambda\left(\mathbf{x}_{0}, t_{0}, \tau\right)$ in the doubly dashed region $B_{i} \cap \Phi^{-1}\left(B_{k}\right)$.
We can use our estimation of $\mathbf{P}\left(t_{0}, \tau\right)_{i k}$ to compute the sum appearing in the network entropies definition Eq. (11). The assumption of uniform FTLE inside region $B_{i} \cap$ $\Phi_{t_{0}+\tau}^{-\tau}\left(B_{k}\right)$ allows us to freely replace functions of $\lambda_{i k}$ by average values in that region

$$
\begin{aligned}
\sum_{k=1}^{N}\left(\mathbf{P}\left(t_{0}, \tau\right)_{i k}\right)^{q} & \approx \sum_{k=1}^{N} e^{-q \lambda_{i k} \tau} \\
& \approx \sum_{k=1}^{N} \frac{1}{\Delta^{2} e^{-\lambda_{i k} \tau}} \int_{B_{i} \cap \Phi_{t_{0}+\tau}^{-\tau}\left(B_{k}\right)} e^{-q \lambda\left(\mathbf{x}_{0}, t_{0}, \tau\right) \tau} d \mathbf{x}_{0} \\
& \approx \sum_{k=1}^{N} \frac{1}{\Delta^{2}} \int_{B_{i} \cap \Phi_{t_{0}+\tau}^{-\tau}\left(B_{k}\right)} e^{(1-q) \lambda\left(\mathbf{x}_{0}, t_{0}, \tau\right) \tau} d \mathbf{x}_{0} \\
& =\frac{1}{\Delta^{2}} \int_{B_{i}} e^{(1-q) \lambda\left(\mathbf{x}_{0}, t_{0}, \tau\right) \tau} d \mathbf{x}_{0},
\end{aligned}
$$

which, using definition (11), implies

$$
e^{(1-q) \tau H_{i}^{q}\left(t_{0}, \tau\right)} \approx\left\langle e^{(1-q) \tau \lambda\left(\mathbf{x}_{0}, t_{0}, \tau\right)}\right\rangle_{B_{i}} .
$$

This is the sought relationship between network entropies and moments of the stretching factor $e^{\lambda \tau}$. For $q=0$, we reobtain Eq. (10). In the limit $q \rightarrow 1$, we get $H_{i}^{1}\left(t_{0}, \tau\right)$ $\approx\left\langle\lambda\left(\mathbf{x}_{0}, t_{0}, \tau\right)\right\rangle_{B_{i}}=\lambda_{i}\left(t_{0}, \tau\right)$. The arguments above can be repeated to get the same relationship (A3) between network entropies in the backwards time direction and backwards Lyapunov exponents.

All these expressions are similar to the ones presented, for example, by Paladin and Vulpiani ${ }^{71}$ relating Rényi entropies and generalized Lyapunov exponents defined from moments of the stretching factor $e^{\lambda \tau}$. But here, the moments are not by averaging along a dynamic trajectory but inside a box $B_{i}$. In the same way, as the value of any of the network entropies at node $i$ characterizes the inhomogeneity in the fluxes sent from $i$ to other nodes, the difference between the different entropies (different $q$ ) at a single node $i$ characterizes the inhomogeneity of the FTLE inside box $B_{i}$. This is a way by which small-scale features present in the Lagrangian trajectories get statistically represented in the network description. Relationships such as (A3) are not exact for finite $\Delta$ and $\tau$, but we expect them to become more accurate for increasing $\tau$ and decreasing $\Delta$.

\section{APPENDIX B: THE INFOMAP METHOD}

Infomap ${ }^{67}$ is a community-detection algorithm ${ }^{43,63-66}$ that retains both the "direction" and "weight" information of each link in the network.

Infomap does not require to specify a priory the number of communities to be detected. It finds structures which are directly related to well-mixed regions under the flow represented by $\mathbf{P}\left(t_{0}, \tau\right)$, and not to other structural properties (for example, a well defined region with strong fluxes oriented towards a particular direction) which will not lead to particle localization in that region. Also, Infomap does not assume communities with similar sizes (as, for example, spectral partitioning ${ }^{16,17}$ ) nor suffers from the "resolution limit",68 which limits the minimum community size detectable by 
most algorithms. In fact, the method decomposes the transport network into subgraphs of different sizes where the flow requires so.

In addition to these convenient properties, the minimization algorithm is efficiently implemented in publicly available software (http://www.tp.umu.se/ rosvall/code.html).

Infomap considers an ensemble of random walkers in the weighted and directed network defined by $\mathbf{P}\left(t_{0}, \tau\right)$, moving with the transition probabilities in that matrix. Then, the method considers from the information-theory point of view the optimal coding of the ensemble of possible random walks. To this end, the network is divided in communities and each random walk is coded by sequences of words that represent successive locations inside a community and jumps between different communities. The information-theoretic lower bound to the average length of the codeword used is given in terms of the transition probabilities and of the specific partition in communities by the so-called map equation

$$
L=q_{\curvearrowright} H(\mathcal{Q})+\sum_{\alpha=1}^{c} p_{\circlearrowright}^{\alpha} H\left(\mathcal{P}^{\alpha}\right) .
$$

$c$ is the number of communities in the particular partition considered. The first term involves the Shanon entropy associated to the transitions between different communities $\alpha$

$$
H(\mathcal{Q})=-\sum_{\alpha=1}^{c} \frac{q_{\alpha \curvearrowright}}{q_{\curvearrowright}} \log _{2}\left(\frac{q_{\alpha \curvearrowright}}{q_{\curvearrowright}}\right) .
$$

$q_{\alpha \curvearrowright}$ is the probability to leave community $\alpha$ in one randomwalk step, and $q_{\curvearrowright}=\sum_{\alpha=1}^{c} q_{\alpha \curvearrowright}$. Expressions for these quantities in terms of the components of the network matrix $\mathbf{P}\left(t_{0}, \tau\right)$ are given in Rosvall and Bergstrom. ${ }^{67}$ The second term in Eq. (B1) contains the Shanon entropies $H\left(\mathcal{P}^{\alpha}\right)$ associated to the words used to codify the position inside a community $\alpha$ and the word that denote the exit from that community

$$
H\left(\mathcal{P}^{\alpha}\right)=-\sum_{i \in \alpha} \frac{\pi_{i}}{p_{\circlearrowright}^{\alpha}} \log _{2}\left(\frac{\pi_{i}}{p_{\circlearrowright}^{\alpha}}\right)-\frac{q_{\alpha \curvearrowright}}{p_{\circlearrowright}^{\alpha}} \log _{2}\left(\frac{q_{\alpha \curvearrowright}}{p_{\circlearrowright}^{\alpha}}\right) .
$$

The notation $i \in \alpha$ indicates sum over the nodes pertaining to community $\alpha . \pi_{i}$ is the stationary distribution of the random walk and $p_{\circlearrowright}^{\alpha}=q_{\alpha \curvearrowright}+\sum_{i \in \alpha} \pi_{i}$. Again, expressions for these quantities can be obtained from the elements in the network matrix $\mathbf{P}\left(t_{0}, \tau\right){ }^{67}$

Infomap finds the partition that minimizes the quantity in (B1), i.e., the partition that provides a shorter description of the ensemble of walks going in and outside the communities. In other words, it finds the partition for which the random walks remain most of the time inside the communities with few jumps between them. This minimization process uses a deterministic greedy algorithm followed by a simulated-annealing which was repeated 100 times to select the best partition in provinces (although the results were already stable after 10 attempts).

${ }^{1}$ V. Rom-Kedar, A. Leonard, and S. Wiggins, "An analytical study of transport, mixing and chaos in an unsteady vortical flow," J. Fluid Mech. 214, 347-394 (1990).
${ }^{2}$ A. Poje and G. Haller, "Geometry of cross-stream mixing in a double-gyre ocean model," J. Phys. Oceanogr. 29, 1649-1665 (1999).

${ }^{3}$ A. M. Mancho, D. Small, and S. Wiggins, "A tutorial on dynamical systems concepts applied to Lagrangian transport in oceanic flows defined as finite time data sets: Theoretical and computational issues," Phys. Rep. 437, 55-124 (2006).

${ }^{4}$ A. Mancho, E. Hernández-García, D. Small, S. Wiggins, and V. Fernández, "Lagrangian transport through an ocean front in the NorthWestern Mediterranean Sea," J. Phys. Oceanogr. 38, 1222-1237 (2008).

${ }^{5} \mathrm{~S}$. Balasuriya, "Explicit invariant manifolds and specialised trajectories in a class of unsteady flows," Phys. Fluids 24, 127101 (2012).

${ }^{6} \mathrm{G}$. Haller and G. Yuan, "Lagrangian coherent structures and mixing in two-dimensional turbulence," Physica D 147, 352-370 (2000).

${ }^{7}$ T. Peacock and J. Dabiri, "Introduction to focus issue: Lagrangian coherent structures," Chaos 20, 017501 (2010).

${ }^{8}$ G. Haller and F. J. Beron-Vera, "Geodesic theory of transport barriers in two-dimensional flows," Physica D 241, 1680-1702 (2012).

${ }^{9} \mathrm{G}$. Haller, "Distinguished material surfaces and coherent structure in three-dimensional fluid flows," Physica D 149, 248-277 (2001).

${ }^{10} \mathrm{~B}$. Joseph and B. Legras, "Relation between kinematic boundaries, stirring, and barriers for the Antartic polar vortex," J. Atmos. Sci. 59, $1198-1212$ (2002).

${ }^{11}$ F. d'Ovidio, V. Fernandez, E. Hernandez-García, and C. Lopez, "Mixing structures in the Mediterranean Sea from finite-size Lyapunov exponents," Geophys. Res. Lett. 31, L17203, doi:10.1029/2004GL020328 (2004).

${ }^{12}$ S. C. Shadden, F. Lekien, and J. E. Marsden, "Definition and properties of Lagrangian coherent structures from finite-time Lyapunov exponents in two-dimensional aperiodic flows," Physica D 212, 271-304 (2005).

${ }^{13}$ F. Huhn, A. von Kameke, S. Allen-Perkins, P. Montero, A. Venancio, and V. Pérez-Muñuzuri, "Horizontal Lagrangian transport in a tidal-driven estuary-Transport barriers attached to prominent coastal boundaries," Cont. Shelf Res. 39-40, 1-13 (2012).

${ }^{14}$ A. M. Mancho, S. Wiggins, J. Curbelo, and C. Mendoza, "Lagrangian descriptors: A method for revealing phase space structures of general time dependent dynamical systems," Commun. Nonlinear Sci. Numer. Simul. 18, 3530-3557 (2013).

${ }^{15} \mathrm{G}$. Froyland and M. Dellnitz, "Detecting and locating near-optimal almost-invariant sets and cycles," SIAM J. Sci. Comput. 24, 1839-1863 (2003).

${ }^{16}$ G. Froyland, "Statistically optimal almost-invariant sets," Physica D 200, 205-219 (2005).

${ }^{17}$ G. Froyland, K. Padberg, M. H. England, and A. M. Treguier, "Detection of coherent oceanic structures via transfer operators," Phys. Rev. Lett. 98, 224503 (2007).

${ }^{18}$ M. Dellnitz, G. Froyland, C. Horenkamp, K. Padberg-Gehle, and A. Sen Gupta, "Seasonal variability of the subpolar gyres in the Southern Ocean: A numerical investigation based on transfer operators," Nonlinear Processes Geophys. 16, 655-663 (2009).

${ }^{19}$ G. Froyland, N. Santitissadeekorn, and A. Monahan, "Transport in timedependent dynamical systems: Finite-time coherent sets," Chaos 20, 043116 (2010).

${ }^{20}$ N. Santitissadeekorn, G. Froyland, and A. Monahan, "Optimally coherent sets in geophysical flows: A transfer-operator approach to delimiting the stratospheric polar vortex," Phys. Rev. E 82, 056311 (2010).

${ }^{21}$ Z. Levnajić and I. Mezić, "Ergodic theory and visualization. I. Mesochronic plots for visualization of ergodic partition and invariant sets," Chaos 20, 033114 (2010).

${ }^{22}$ G. Froyland, C. Horenkamp, V. Rossi, N. Santitissadeekorn, and A. S. Gupta, "Three-dimensional characterization and tracking of an Agulhas ring," Ocean Modell. 52, 69-75 (2012).

${ }^{23} \mathrm{P}$. Tallapragada and S. D. Ross, "A set oriented definition of finite-time Lyapunov exponents and coherent sets," Commun. Nonlinear Sci. Numer. Simul. 18, 1106-1126 (2013).

${ }^{24}$ F. Lekien, S. C. Shadden, and J. E. Marsden, "Lagrangian coherent structures in n-dimensional systems," J. Math. Phys. 48, 065404 (2007).

${ }^{25}$ G. Froyland and K. Padberg, "Almost-invariant sets and invariant manifolds-connecting probabilistic and geometric descriptions of coherent structures in flows," Physica D 238, 1507-1523 (2009).

${ }^{26} \mathrm{M}$. Branicki and S. Wiggins, "Finite-time Lagrangian transport analysis: Stable and unstable manifolds of hyperbolic trajectories and finite-time Lyapunov exponents," Nonlinear Processes Geophys. 17, 1-36 (2010).

${ }^{27}$ G. Haller, "A variational theory of hyperbolic Lagrangian coherent structures," Physica D 240, 574-598 (2011); M. Farazmand and G. Haller, "Erratum and addendum to "A variational theory of hyperbolic Lagrangian 
coherent structures (Physica D 240 (2011) 574-598)'," Physica D 241, 439-441 (2012).

${ }^{28} \mathrm{D}$. Karrasch, "Comment on "A variational theory of hyperbolic Lagrangian coherent structures (Physica D 240 (2011) 574-598)'," Physica D 241, 1470-1473 (2012).

${ }^{29}$ G. Froyland and K. Padberg-Gehle, "Finite-time entropy: A probabilistic approach for measuring nonlinear stretching," Physica D 241, 1612-1628 (2012).

${ }^{30}$ D. W. Waugh and E. R. Abraham, "Stirring in the global surface ocean," Geophys. Res. Lett. 35, L20605, doi:10.1029/2008GL035526 (2008).

${ }^{31}$ V. Rossi, C. López, E. Hernández-García, J. Sudre, V. Garçon, and Y. Morel, "Surface mixing and biological activity in the four Eastern Boundary upwelling systems," Nonlinear Processes Geophys. 16, 557-568 (2009).

${ }^{32}$ I. Hernández-Carrasco, C. López, E. Hernández-García, and A. Turiel, "Seasonal and regional characterization of horizontal stirring in the global ocean,” J. Geophys. Res. 117, C10007, doi:10.1029/2012JC008222 (2012).

${ }^{33}$ R. Preis, M. Dellnitz, M. Hessel, C. Schütte, and E. Meerbach, "Dominant paths between almost invariant sets of dynamical systems," preprint 154 of the DFG Schwerpunktprogramm 1095 (2004), see http://www2.math.uni-paderborn.de/ags/ag-dellnitz.

${ }^{34}$ M. Dellnitz, M. Hessel-von Molo, P. Metzner, R. Preis, and C. Schütte, "Graph algorithms for dynamical systems," in Analysis, Modeling and Simulation of Multiscale Problems, edited by A. Mielke (Springer Verlag, Heidelberg, 2006), pp. 619-645.

${ }^{35} \mathrm{~N}$. Santitissadeekorn and E. Bollt, "Identifying stochastic basin hopping by partitioning with graph modularity," Physica D 231, 95-107 (2007).

${ }^{36} \mathrm{M}$. Nilsson-Jacobi, C. André, K. Doos, and P. R. Jonsson, "Identification of subpopulations from connectivity matrices," Ecography 35, 1004-1016 (2012).

${ }^{37}$ M. Speetjens, M. Lauret, H. Nijmeijer, and P. Anderson, "Footprints of Lagrangian flow structures in Eulerian concentration distributions in periodic mixing flows," Physica D 250, 20-33 (2013).

${ }^{38}$ C. J. Thomas, J. Lambrechts, E. Wolanski, V. Traag, V. D. Blondel, E. Deleersnijder, and E. Hanert, "Numerical modelling and graph theory tools to study ecological connectivity in the Great Barrier Reef," Ecol. Modell. 272, 160-174 (2014).

${ }^{39}$ V. Rossi, E. Ser-Giacomi, C. López, and E. Hernández-García, "Hydrodynamic provinces and oceanic connectivity from a transport network help designing marine reserves," Geophys. Res. Lett. 41, 2883-2891, doi:10.1002/2014GL059540 (2014).

${ }^{40}$ R. Albert and A.-L. Barabási, "Statistical mechanics of complex networks," Rev. Mod. Phys. 74, 47-97 (2002).

${ }^{41}$ S. N. Dorogovtsev and J. F. F. Mendes, "Evolution of networks," Adv. Phys. 51, 1079-1187 (2002).

${ }^{42}$ S. Boccaletti, V. Latora, Y. Moreno, M. Chavez, and D.-U. Hwang, "Complex networks: Structure and dynamics," Phys. Rep. 424, 175-308 (2006).

${ }^{43}$ M. E. J. Newman, Networks: An Introduction (Oxford University Press, Oxford, 2010).

${ }^{44}$ A. A. Tsonis, K. L. Swanson, and P. J. Roebber, "What do networks have to do with climate?,” Bull. Am. Meteorol. Soc. 87, 585-595 (2006).

${ }^{45} \mathrm{~K}$. Yamasaki, A. Gozolchiani, and S. Havlin, "Climate networks around the globe are significantly affected by El Niño," Phys. Rev. Lett. 100, 228501 (2008).

${ }^{46}$ J. F. Donges, Y. Zou, N. Marwan, and J. Kurths, "The backbone of the climate network," EPL (Europhys. Lett.) 87, 48007 (2009).
${ }^{47}$ N. Malik, B. Bookhagen, N. Marwan, and J. Kurths, "Analysis of spatial and temporal extreme monsoonal rainfall over South Asia using complex networks," Climate Dyn. 39, 971-987 (2012).

${ }^{48}$ A. Gozolchiani, S. Havlin, and K. Yamasaki, "Emergence of El Niño as an autonomous component in the climate network," Phys. Rev. Lett. 107, 148501 (2011).

${ }^{49}$ M. Barreiro, A. C. Marti, and C. Masoller, "Inferring long memory processes in the climate network via ordinal pattern analysis," Chaos 21, 013101 (2011).

${ }^{50}$ Y. Berezin, A. Gozolchiani, O. Guez, and S. Havlin, "Stability of climate networks with time," Sci. Rep. 2, 666 (2012).

${ }^{51}$ N. Molkenthin, K. Rehfeld, N. Marwan, and J. Kurths, "Networks from flows-from dynamics to topology," Sci. Rep. 4, 4119 (2014).

${ }^{52}$ M. E. Newman, "Analysis of weighted networks," Phys. Rev. E 70, 056131 (2004).

${ }^{53}$ M. Barthélemy, “Spatial networks," Phys. Rep. 499, 1-101 (2011).

${ }^{54}$ P. Holme and J. Saramäki, "Temporal networks," Phys. Rep. 519, 97-125 (2012).

${ }^{55}$ G. Froyland, R. M. Stuart, and E. van Sebille, "How well-connected is the surface of the global ocean?," Chaos 24, 033126 (2014).

${ }^{56}$ P. Oddo, M. Adani, N. Pinardi, C. Fratianni, M. Tonani, D. Pettenuzzo et al., "A nested Atlantic-Mediterranean Sea general circulation model for operational forecasting," Ocean Sci. 5, 461 (2009).

${ }^{57}$ C. Millot and I. Taupier-Letage, "Circulation in the Mediterranean Sea," in The Mediterranean Sea, Handbook of Environmental Chemistry Vol. 5K, edited by A. Saliot (Springer-Verlag, Berlin, Heidelberg, 2005), pp. 29-66.

${ }^{58}$ T. Tél and M. Gruiz, Chaotic Dynamics: An Introduction Based on Classical Mechanics (Cambridge University Press, Cambridge, 2006).

${ }^{59}$ A. Rényi, Probability Theory (North-Holland, Amsterdam, 1970).

${ }^{60}$ G. Boffetta, M. Cencini, M. Falcioni, and A. Vulpiani, "Predictability: A way to characterize complexity," Phys. Rep. 356, 367-474 (2002).

${ }^{61} \mathrm{P}$. Castiglione, M. Falcioni, A. Lesne, and A. Vulpiani, Chaos and Coarse Graining in Statistical Mechanics (Cambridge University Press, Cambridge, 2010).

${ }^{62}$ M. Cencini, F. Cecconi, and A. Vulpiani, Chaos: From Simple Models to Complex Systems (World Scientific, Singapore, 2010).

${ }^{63}$ L. Danon, A. Díaz-Guilera, J. Duch, and A. Arenas, "Comparing community structure identification,” J. Stat. Mech. 09, P09008 (2005).

${ }^{64}$ A. Lancichinetti and S. Fortunato, "Community detection algorithms: A comparative analysis,” Phys. Rev. E 80, 056117 (2009).

${ }^{65}$ S. Fortunato, "Community detection in graphs," Phys. Rep. 486, 75-174 (2010).

${ }^{66} \mathrm{R}$. Aldecoa and I. Marín, "Exploring the limits of community detection strategies in complex networks," Sci. Rep. 3, 2216 (2013).

${ }^{67}$ M. Rosvall and C. T. Bergstrom, "Maps of random walks on complex networks reveal community structure," Proc. Natl. Acad. Sci. U.S.A. 105, $1118-1123$ (2008).

${ }^{68} \mathrm{~S}$. Fortunato and M. Barthélemy, "Resolution limit in community detection," Proc. Natl. Acad. Sci. U.S.A. 104, 36-41 (2007).

${ }^{69} \mathrm{M}$. Schaub, R. Lambiotte, and M. Barahona, "Encoding dynamics for multiscale community detection: Markov time sweeping for the map equation," Phys. Rev. E 86, 026112 (2012).

${ }^{70}$ M. E. Newman and M. Girvan, "Finding and evaluating community structure in networks," Phys. Rev. E 69, 026113 (2004).

${ }^{71}$ G. Paladin and A. Vulpiani, "Anomalous scaling laws in multifractal objects," Phys. Rep. 156, 147-225 (1987). 